\title{
Observable Emission Features of Black Hole GRMHD Jets on Event Horizon Scales
}

\author{
Hung-Yi $\mathrm{Pu}^{1,2}$, Kinwah $\mathrm{Wu}^{3,4}$, Ziri Younsi ${ }^{5}$, Keiichi Asada ${ }^{2}$, Yosuke Mizuno ${ }^{5}$ (1), and Masanori Nakamura ${ }^{2}$ (1) \\ ${ }^{1}$ Perimeter Institute for Theoretical Physics, 31 Caroline Street North, Waterloo, ON, N2L 2Y5, Canada; hpu@ perimeterinstitute.ca \\ ${ }^{2}$ Institute of Astronomy \& Astrophysics, Academia Sinica, 11F of Astronomy-Mathematics Building, AS/NTU No. 1, Taipei 10617, Taiwan \\ asada@asiaa.sinica.edu.tw, nakamura@asiaa.sinica.edu.tw \\ ${ }^{3}$ Mullard Space Science Laboratory, University College London, Holmbury St. Mary, Dorking, Surrey, RH5 6NT, UK; kinwah.wu@ucl.ac.uk \\ ${ }^{4}$ School of Physics, University of Sydney, Sydney, NSW 2006, Australia \\ ${ }^{5}$ Institut für Theoretische Physik, Max-von-Laue-Straße 1, D-60438 Frankfurt am Main, Germany; younsi@th.physik.uni-frankfurt.de, \\ mizuno@th.physik.uni-frankfurt.de \\ Received 2017 May 29; revised 2017 July 17; accepted 2017 July 18; published 2017 August 22
}

\begin{abstract}
The general-relativistic magnetohydrodynamical (GRMHD) formulation for black hole-powered jets naturally gives rise to a stagnation surface, where inflows and outflows along magnetic field lines that thread the black hole event horizon originate. We derive a conservative formulation for the transport of energetic electrons, which are initially injected at the stagnation surface and subsequently transported along flow streamlines. With this formulation the energy spectra evolution of the electrons along the flow in the presence of radiative and adiabatic cooling is determined. For flows regulated by synchrotron radiative losses and adiabatic cooling, the effective radio emission region is found to be finite, and geometrically it is more extended along the jet central axis. Moreover, the emission from regions adjacent to the stagnation surface is expected to be the most luminous as this is where the freshly injected energetic electrons are concentrated. An observable stagnation surface is thus a strong prediction of the GRMHD jet model with the prescribed non-thermal electron injection. Future millimeter/submillimeter $(\mathrm{mm} / \mathrm{sub}-\mathrm{mm})$ very-long-baseline interferometric observations of supermassive black hole candidates, such as the one at the center of M87, can verify this GRMHD jet model and its associated non-thermal electron injection mechanism.
\end{abstract}

Key words: black hole physics - gravitation - galaxies: individual (M87) - galaxies: jets - Galaxy: center radiation mechanisms: non-thermal

\section{Introduction}

It is a pressing question as to how the radiation that is observed in relativistic jets in active galactic nuclei (AGNs) is generated (e.g., Blandford \& Königl 1979; Marscher 1980; Zensus 1997; Laing \& Bridle 2002; Honda 2010; Levinson \& Rieger 2011; Mościbrodzka et al. 2011; Ito et al. 2013; Mason et al. 2013; Potter \& Cotter 2013; Hovatta et al. 2014; Scott \& Stewart 2014; Shih \& Stockton 2014; Wang et al. 2014; Turner \& Shabala 2015; Asada et al. 2016; Hirotani et al. 2016; Koay et al. 2016; Khabibullin et al. 2016; Prieto et al. 2016). Although there is a common consensus that the emitters are energetic particles, how these particles are accelerated to such high energies, how they dissipate their energy, and how they are transported with the jets themselves are still the subject of feverish investigation (e.g., Blandford \& Eichler 1987). It is argued that relativistic outflows from black holes are associated with accretion flows (Blandford 1976; Fender et al. 2004; Meier 2005; Ferreira et al. 2006; Trump et al. 2011; Pu et al. 2012; Wu et al. 2013; Ishibashi et al. 2014; Sbarrato et al. 2014). In the case of collimated relativistic jets, magnetic fields must play an important role (Camenzind 1986a, 1986b, 1987; Fendt \& Greiner 2001; Vlahakis \& Königl 2004; Komissarov et al. 2007; Lyubarsky 2009; Nakamura \& Asada 2013; Homan et al. 2015), and it is argued that jets are powered at the expense of the black hole, wherein energy is extracted from a reservoir of rotational energy from the black hole itself, either by electromagnetic means (Blandford \& Znajek 1977; Komissarov 2004, 2005; Toma \& Takahara 2014) or through magnetohydrodynamical processes (Phinney 1983; Takahashi et al. 1990; Koide et al. 2002; McKinney \& Gammie 2004; Hawley \& Krolik 2006). Models have been proposed for both of these cases, and they both in principle possess certain testable predictions. In particular, for the latter, numerical GRMHD simulations (e.g., McKinney 2006) and analytical GRMHD studies (e.g., Takahashi et al. 1990; Pu et al. 2016) consistently show the presence of a stagnation or separation surface (a separatrix). This surface separates the (inner) inflow region from the (outer) outflow region, both of which follow the same global, black-hole-threading magnetic field lines. The relatively slow radial velocities near the stagnation surface imply a high concentration of fluid particles. If energetic particles are injected in the vicinity of the stagnation surface or near the black hole event horizon, they must accumulate in high concentrations near the stagnation surface, provided that the cooling timescale is not significantly shorter than the dynamical timescale of the jet fluid flow. This surface is a unique feature of relativistic GRMHD jets and in contrast to an ideal force-free magnetic jet (e.g., McKinney \& Narayan 2007; Tchekhovskoy et al. 2008; Broderick \& Loeb 2009).

In a GRMHD jet model (e.g., Camenzind 1986b; Takahashi et al. 1990), the fluid mass mainly comprises baryons, although this does not rule out the possibility that there could also be a substantial number of positively charged leptons. For such a baryonic jet, one would expect that the $\mathrm{mm} / \mathrm{sub}-\mathrm{mm}$ radio emission is predominantly synchrotron radiation from energetic leptons, which are likely non-thermal in origin. In a simple approximation, the radiative power of leptonic synchrotron emission is related to the magnetic energy density as $P \propto \bar{\gamma}^{2} \bar{\beta}^{2} B^{2}$, where $\bar{\gamma}$ is the effective Lorentz factor, $\bar{\beta}$ is the effective velocity of the leptons (normalized to the speed of light), and $B$ is the magnetic field strength. While the non-thermal synchrotron model is in general consistent with polarimetric and spectral observations of the radio 
emission from AGN jets (e.g., Broderick \& McKinney 2010; Clausen-Brown et al. 2011), the exact origin of non-thermal particles (e.g., electrons in the context of baryonic jets) is still an unresolved issue.

In this study, we aim to determine the observational signatures of the stagnation surface presented in model GRMHD jets in the context of non-thermal synchrotron radiation from baryon-dominated relativistic flows. In spite of the many uncertainties in the detailed microphysics, many of these are unimportant in determining the qualitative aspects of the observable features. Due to the absence of physical models for the injection of non-thermal electrons into jets, certain assumptions have been adopted in the pioneering works in the field, such as phenomenological mass-loading models (Broderick \& Loeb 2009) or a fixed ratio of the internal energy of non-thermal electrons to the magnetic energy (Dexter et al. 2012). These assumptions have restricted the "thermal properties" (such as the the energy cutoff and the spectral index) of the non-thermal synchrotron-emitting particles to be rigidly associated with the jet flow. We relax such restrictions by allowing the energy content of the particles to evolve as they are transported in the flow through appropriate loss processes, such as synchrotron and adiabatic processes. This improvement enables us to provide more reliable observational signatures for the GRMHD jet. With forthcoming $\mathrm{mm} / \mathrm{sub}-\mathrm{mm}$ VLBI observations of AGN jets, in particular the one in M87 by the Event Horizon Telescope (Doeleman et al. 2012; Lu et al. 2014; Kino et al. 2015; Ricarte \& Dexter 2015) and the Greenland Telescope (GLT; Nakamura et al. 2013; Inoue et al. 2014; Raffin et al. 2016), definitive observable emission signatures like those presented in this work will prove essential in testing theoretical models.

The paper is organized as follows. The modeling of the spatial and energy population of non-thermal electrons along a background GRMHD flow is presented in Section 2. A model to qualitatively describe a GRMHD flow is described in Section 3. The results and implications of the spatial variation of non-thermal electrons are given in Section 4. Finally, the summary and conclusions are presented in Section 5.

\section{Spatial and Energy Population of Non-thermal Electrons}

In this section, we formulate the population of non-thermal energetic particles and their evolution in a stationary jet flow. As the flow within the jet follows streamlines, the particle number density along each flow streamline evolves from an initial value. ${ }^{6}$ This enables the construction of a simple analytic spectral evolutionary model for the non-thermal particles along the flow within the entire jet, provided that (i) the non-thermal

\footnotetext{
6 In a one-zone formulation, the energy spectral evolution of a population of energetic particles is governed by the transport equation

$$
\frac{\partial n(\gamma)}{\partial t}+\frac{\partial}{\partial \gamma} \dot{\gamma} n(\gamma)=\dot{Q}_{\text {inj }}(\gamma)-\dot{Q}_{\text {loss }}(\gamma)
$$

(see, e.g., Ginzburg \& Syrovatskii 1964), where $n(\gamma)$ is the particle number density, and $\dot{Q}_{\text {inj }}$ and $\dot{Q}_{\text {loss }}$ are the particle injection and particle loss rates, respectively. This equation has assumed that the angular distribution of the particles in momentum space is preserved, which is justified in a magnetically confined region if radiative losses are insignificant. In the MHD jet model considered here, the energetic (non-thermal) particles are carried along the flow streamlines (which are parallel to the magnetic field lines) by the fluid. This formulation is therefore not applicable. A covariant formulation that takes into account the spatial transport is required when constructing the transport equation for the energy spectral evolution of the particle population (see, e.g., Chael et al. 2017).
}

particles do not thermalize on timescales shorter than the dynamical timescale of the flow, (ii) there is no further injection and no leakage of particles along the flow, and the diffusion of non-thermal particles across flow streamlines is negligible, and (iii) collisions between non-thermal particles, and the scattering and drift along magnetic field lines (and flow streamlines) are unimportant. In a stationary state, the evolution of the nonthermal particle spectral density along a specific flow streamline can therefore be expressed as

$$
n_{\text {nth }}(\gamma, s)=\left.\lambda_{0} n_{\text {th }}(s) \mathcal{G}(\gamma)\right|_{s},
$$

where $s$ is the poloidal location on the flow streamline, $\lambda_{0}$ is a parameter specifying the relative fraction of the non-thermal particles, and $\mathcal{G}(\gamma)$ is the normalized energy spectral distribution function of the non-thermal particles, evaluated in the comoving frame of the bulk flow. The absence of particle injection, leakage, and thermalization implies that $\lambda_{0}$ will remain constant along each flow streamline. The number of thermal particles is given by the density of the fluid in the flow, which is the solution of the GRMHD equations of the jet.

\subsection{Transport of the Particle Distribution Function}

Note that Liouville's theorem for the conservation of phasespace volume along geodesics is not applicable to determine $\mathcal{G}(\gamma)$, as it is not a locally or globally free-falling system. More specifically, the flow of non-thermal radiating particles (presumably electrons) in the configuration space is constrained by the bulk motion of MHD fluid in the jet, and the flow in the momentum space is determined by the radiative loss and the thermo-hydrodynamics of the jet fluid. We may, however, formulate the transport equation to determine the profile of $\left.\mathcal{G}(\gamma)\right|_{s}$ along $s$ for the non-thermal particles in terms of the particles' cumulative energy spectral distribution function by taking advantage of the probability invariance and the particle number conservation along the flow.

We start by defining the cumulative energy spectral distribution function of the non-thermal particles, evaluated at location $s$, as

$$
\mathcal{F}(\gamma, s)=\left.\int_{1}^{\gamma} d \gamma^{\prime} \mathcal{G}\left(\gamma^{\prime}\right)\right|_{s},
$$

whose normalization is

$$
\left.\int_{1}^{\gamma_{\max }} d \gamma \mathcal{G}(\gamma)\right|_{s}=1 .
$$

This cumulative energy distribution function $\mathcal{F}(\gamma, s)$ is essentially the area under the curve $\left.\mathcal{G}\left(\gamma^{\prime}\right)\right|_{s}$, bounded by $\gamma^{\prime}=1$ and $\gamma$.

In the absence of particle scattering, the condition $\gamma_{\mathrm{b}}>\gamma_{\mathrm{a}}$ is invariant along $s$ for any arbitrary pair of particles "a" and "b". This ensures that $\mathcal{F}\left(\gamma_{2}, s_{2}\right)=\mathcal{F}\left(\gamma_{1}, s_{1}\right)$ with only the bounds to determine $\mathcal{F}(\gamma, s)$ being changed from $\gamma_{1}$ to $\gamma_{2}$, when a population of particles located at $s_{1}$ is transported along the characteristic curve to a new location $s_{2}$ (see illustration in Figure 1). The invariance of $\mathcal{F}(\gamma, s)$ along $s$ implies that the cumulative distribution function satisfies the advective transport equation

$$
\left[\frac{\partial}{\partial s}+\frac{d \gamma}{d s} \frac{\partial}{\partial \gamma}\right] \mathcal{F}(\gamma, s)=0
$$




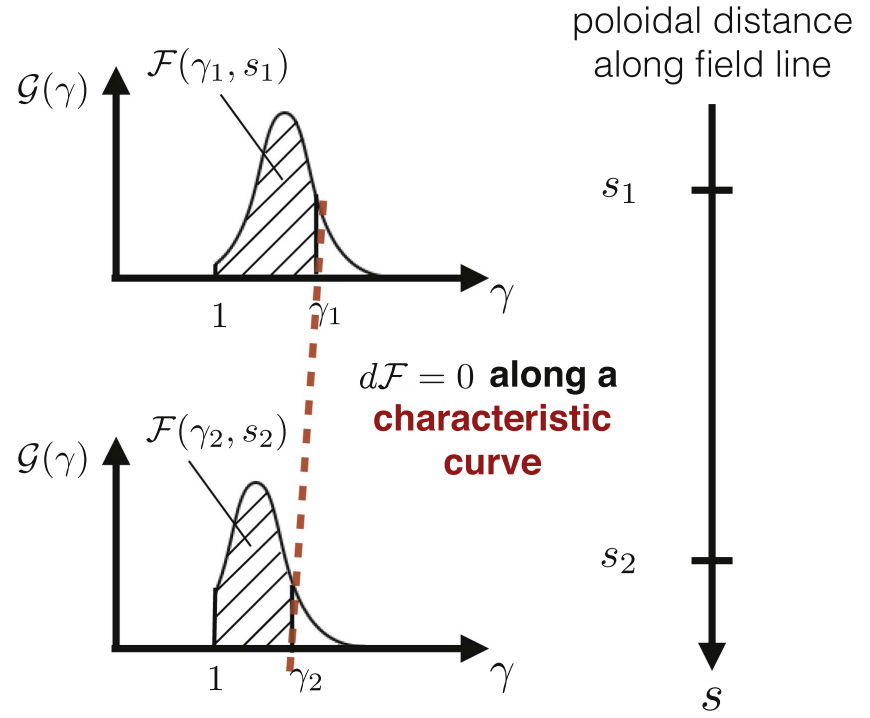

Figure 1. Illustration of tracing the spatial variation of the non-thermal electron energy, $\gamma$, by using $\left.\mathcal{G}(\gamma)\right|_{s}$ (see Section 2.1) and a moving boundary such that $d \mathcal{F}=0$ (red dashed line). $\mathcal{F}(\gamma, s)$ is the cumulative energy spectral distribution function (Equation (2)), represented by the shaded region. The moving boundary $d \mathcal{F}=0$ can be described by a characteristic curve (Equation (5)). See Section 2.1 for details.

and $\mathcal{G}(\gamma)$ is traced by the curves $d \mathcal{F}=0$, which are the characteristic curves. Hence, the transport equation can be solved using the method of characteristics once the energy gain/loss rate along $s, d \gamma / d s$, is specified.

In the reference frame co-moving with the bulk motion of the transported jet fluid,

$$
\frac{d \gamma}{d s}=\left.\frac{\dot{\gamma}}{v} \frac{d \tau}{d t}\right|_{s}
$$

where $d \tau$ is the proper time interval, and $\dot{\gamma}(\equiv d \gamma / d \tau)$ is the energy gain/loss per unit time. Since $s$ is increasing from the black hole to infinity, we can freely choose where $s=0$. Here and hereafter, we adopt the convention $s=s_{0}=0$ at the stagnation surface. Therefore, $d s>0$ for the outflow and $d s<0$ for the inflow in the jet. The speed of the jet fluid, $v=d s / d t$, may then be written (as measured in the BoyerLindquist coordinate frame) as

$$
v=\sqrt{\frac{\Sigma}{\Delta}}\left[v^{r}+v^{\theta}\left(\frac{d r}{d \theta}\right)\right] \sqrt{1+\Delta\left(\frac{d \theta}{d r}\right)^{2}},
$$

where $\Sigma \equiv r^{2}+a^{2} \cos ^{2} \theta, \Delta \equiv r^{2}-2 M_{\mathrm{BH}} r+a^{2}, M_{\mathrm{BH}}$ is the black hole mass, $a$ is the spin parameter of the black hole, $v^{i} \equiv u^{i} / u^{t}$, and $u^{\mu}$ is the four-velocity of the fluid. The expression for $d r / d \theta$ in the above equation is given by the stream function (Section 3).

For non-thermal particles with $\mathcal{F}\left(\gamma, s_{0}\right)$ injected at $s=s_{0}$ in a stationary manner, the transport equation may now be expressed as

$$
\left[\frac{\partial}{\partial s}+\frac{d \gamma}{d s} \frac{\partial}{\partial \gamma}\right] \mathcal{F}(\gamma, s)=\mathcal{F}\left(\gamma, s_{0}\right) \delta\left(s-s_{0}\right)
$$

Since freshly accelerated particles in high-energy relativistic systems tend to have a power-law energy spectrum, we simply adopt a power-law spectrum for $\mathcal{F}\left(\gamma, s_{0}\right)$ to avoid unnecessary complications. Hence, we have

$$
\left.\mathcal{G}(\gamma)\right|_{s_{0}} \propto \gamma_{0}^{-\alpha},
$$

where $\left.\gamma_{0} \equiv \gamma\right|_{s_{0}}$. The value of the spectral index $\alpha$ depends on the particle acceleration process (e.g., Bell 1978; Blandford \& Eichler 1987; Sironi \& Spitkovsky 2011, 2014; Werner et al. 2016). The characteristic curve, which pivots about $\left(\gamma_{0}, s_{0}\right)$, is determined from Equation (5) and is hence independent of $\alpha$, despite the fact that $\mathcal{F}\left(\gamma_{0}, s_{0}\right)$ (Equation (2)) is dependent on the chosen value of $\alpha$.

\subsection{Energy Loss}

The energy losses of relativistic electrons in jet outflows are often attributed to cooling due to synchrotron radiation, Compton scattering, and/or adiabatic volume expansion. The first two are radiative processes determined by the microscopic properties of the particles, and the last one is associated with the hydrodynamics of the bulk outflow of the transported jet fluid. The change in energy of the energetic particles can be expressed as

$$
\begin{aligned}
\dot{\gamma} & =\dot{\gamma}_{\text {rad }}+\dot{\gamma}_{\text {adi }} \\
& =\left(\dot{\gamma}_{\text {syn }}+\dot{\gamma}_{\text {com }}\right)+\dot{\gamma}_{\text {adi }}
\end{aligned}
$$

in both the inflow and outflow regions. In this expression, the radiative loss terms due to synchrotron radiation and Compton scattering ( $\dot{\gamma}_{\text {syn }}$ and $\dot{\gamma}_{\text {com }}$, respectively) are always negative, but the mechanical term due to the adiabatic change in the volume of the fluid element $\left(\dot{\gamma}_{\text {adi }}\right)$ can be positive (for compression heating) or negative (for expansion cooling).

The radiative losses for relativistic electrons due to synchrotron radiation or Compton scattering (assuming the electrons have an isotropic momentum distribution and energy specified by a Lorentz factor $\gamma$ ) are given (Tucker 1975) by

$$
\begin{aligned}
\dot{\gamma}_{\text {rad }} & =-\frac{4}{3} \frac{\sigma_{\mathrm{T}}}{m_{\mathrm{e}} c}\left(\gamma^{2}-1\right) U_{\mathrm{x}} \\
& \approx-3.2 \times 10^{-8}\left(\gamma^{2}-1\right) U_{\mathrm{x}} \mathrm{s}^{-1} .
\end{aligned}
$$

Here, $\sigma_{\mathrm{T}}$ is the Thomson scattering cross-section and $U_{\mathrm{x}}$ is the energy density of the magnetic field $\left(U_{\mathrm{x}}=U_{\mathrm{mag}}=B^{2} / 8 \pi\right)$ for synchrotron radiation, which is also the energy density of the photon radiation field $\left(U_{\mathrm{x}}=U_{\mathrm{ph}}\right)$ for Compton scattering.

No energy or particles (which transport kinetic energy) leak from this volume element $(\delta V)$ in a strictly adiabatic process. Hence, the energy gain/loss of the particles caused by an adiabatic increase/decrease in volume is

$$
\dot{\gamma}_{\text {adi }} \approx-\left.\frac{(\gamma-1)}{\delta V} \frac{d(\delta V)}{d \tau}\right|_{\text {nth }} \mathrm{s}^{-1},
$$

where $\left.\delta V\right|_{\text {nth }}$ is a co-moving volume element containing a specific number of energetic electrons. As the diffusion drift of non-thermal particles is unimportant on the timescales of interest, the particle transport is dominated by advection. It is therefore sufficient to set $\left.\delta V\right|_{\text {nth }}=\left.\delta V\right|_{\text {fluid. }}$. Thus, a volume expansion in the fluid $\left(\Delta\left(\left.\delta V\right|_{\text {fluid }}\right)>0\right)$ gives a negative $\dot{\gamma}_{\text {adi }}$ (i.e., cooling) and a volume contraction in the fluid $\left(\Delta\left(\left.\delta V\right|_{\text {fluid }}\right)<0\right)$ gives a positive $\dot{\gamma}_{\text {adi }}$ (i.e., heating).

We may express the time evolution of the jet fluid volume in terms of the time evolution of the mass density of the jet fluid along the flow streamline (since the contribution to the total 
fluid mass by non-thermal particles is negligible), $d \ln \rho=-d \ln (\delta V)$, and obtain

$$
\left.\frac{1}{\delta V} \frac{d(\delta V)}{d \tau}\right|_{\text {fluid }}=-v\left(\frac{d t}{d \tau}\right)\left[\frac{d}{d s} \ln \left(\frac{\rho}{\rho_{0}}\right)\right] \mathrm{s}^{-1},
$$

where $\rho_{0}$ is the initial value of $\rho$ at the stagnation surface, which is introduced to keep the argument of the logarithm function dimensionless. It follows that

$$
\dot{\gamma}_{\text {adi }} \approx(\gamma-1) v\left(\frac{d t}{d \tau}\right)\left[\frac{d}{d s} \ln \left(\frac{\rho}{\rho_{0}}\right)\right] \mathrm{s}^{-1},
$$

an expression valid for both jet inflow and jet outflow. As a result, whether the adiabatic process results in a cooling or heating process is determined by the profile of $\rho$ along the flow. If the profile of $\rho$ is decreasing (or increasing) from the stagnation surface, adiabatic cooling (heating) will take place. We demonstrate that adiabatic cooling occurs both for GRMHD outflows and for GRMHD inflows in Section 4.

Note that $\dot{\gamma}_{\text {rad }}$ is determined by the internal thermodynamical properties of the flow, and hence does not depend explicitly on the geometrical extent of the system. However, $\dot{\gamma}_{\text {adi }}$ is determined by the global thermodynamical properties of the system and is hence expected to scale with the black hole mass, $M_{\mathrm{BH}}$. From the ratio of Equations (10) and (11), we obtain

$$
\left.\frac{\dot{\gamma}_{\text {rad }}}{\dot{\gamma}_{\text {adi }}} \propto \frac{(\gamma+1) U_{\mathrm{x}}(\delta V)}{\mathrm{d}(\delta \mathrm{V}) / \mathrm{d} \tau} \sim t_{\mathrm{dyn}}\right|_{\left\{\gamma, U_{\mathrm{x}}\right\}},
$$

where $t_{\text {dyn }}$ is the dynamical timescale of the flow. For a relativistic jet flow near the black hole event horizon, $t_{\mathrm{dyn}} \sim r_{\mathrm{g}} / c \propto M_{\mathrm{BH}}$, where $r_{\mathrm{g}} \equiv G M_{\mathrm{BH}} / c^{2}$ is the gravitational radius of the black hole. Thus, one would expect that the relative importance of the the radiative cooling and adiabatic cooling would vary according to the black hole mass. In a synchrotron cooling flow, i.e., $U_{\mathrm{x}}=B^{2} / 8 \pi$, if the spatial structure of the magnetic field $B$ is specified and fixed, systems with a more massive black hole would be dominated by synchrotron cooling. In systems with a less massive black hole, adiabatic cooling would become more important.

\subsection{Spatial Variation of $\mathcal{G}(\gamma)$ and Its Dependence on $\alpha$}

The spatial variation of the power law for $d(\ln \mathcal{G}) / d(\ln \gamma)$ at a position $s$ can be approximated by taking $\left(\gamma^{2}-1\right) \approx \gamma^{2}$ and $(\gamma-1) \approx \gamma$ for the energy-loss terms, which is justified when considering only the high-energy component of the electron population. With these approximations, the synchrotron cooling in Equation (5) has the form $d \gamma / d s \propto \gamma^{2}$ and may be written as

$$
\frac{d \gamma}{\gamma^{2}}=-\xi(s) d s
$$

where

$$
\left.\xi(s) \equiv \frac{\sigma_{\mathrm{T}}}{6 \pi m_{\mathrm{e}} c}\left(\frac{B^{2}}{v} \frac{d \tau}{d t}\right)\right|_{s} .
$$

The relation

$$
\gamma_{0}=\frac{\gamma}{1-\gamma x}
$$

is then obtained after some algebra, where

$$
x(s) \equiv \int d s \xi(s)>0 .
$$

Due to the conservation of area on the " $\mathcal{G}(\gamma)-\gamma$ " plane, the relation

$$
\begin{aligned}
\left.\mathcal{G}(\gamma)\right|_{s} d \gamma & =\left.\mathcal{G}(\gamma)\right|_{s_{0}} d \gamma_{0} \\
& =\mathcal{G}_{0} \gamma_{0}^{-\alpha} d \gamma_{0}
\end{aligned}
$$

holds, which upon inserting $d \gamma_{0} / d \gamma$ yields

$$
\left.\mathcal{G}(\gamma)\right|_{s}=\frac{\mathcal{G}_{0} \gamma^{-\alpha}}{(1-\gamma x)^{-\alpha+2}} .
$$

The slope at a given position $s$ is therefore

$$
\left.\frac{d(\ln \mathcal{G})}{d(\ln \gamma)}\right|_{s}=-\alpha-(\alpha-2) x \gamma_{0} .
$$

Since $\alpha>0$, from this relation it may be verified that, at the high-energy end, $\left.[d(\ln \mathcal{G}) / d(\ln \gamma)]\right|_{s}$ is always negative if $\alpha \geqslant 2$. When $\alpha>2$, the highest energy end may have a positive slope, resulting in a "raised-up" profile. An approximation and result similar to that derived here has previously been applied to estimate the time-dependent electron distribution with the form $d \gamma / d t \propto \gamma^{2}$ in Pacholczyk (1970).

In a similar manner, one may repeat this for the adiabatic cooling (Equation (13)) with the form $d \gamma / d s \propto \gamma$, yielding

$$
\left.\frac{d(\ln \mathcal{G})}{d(\ln \gamma)}\right|_{s}=-\alpha
$$

Unlike synchrotron cooling, which has the form $d \gamma / d s \propto \gamma^{2}$, the slope variation due to adiabatic cooling is always negative. Furthermore, the initial slope $-\alpha$ is preserved at different spatial locations,

\section{Flow in an Axisymmetric and Stationary GRMHD Jet}

To investigate the energy variation of non-thermal electrons within a background GRMHD flow, we proceed to construct a "qualitatively correct" GRMHD flow configuration as illustrated in Figure 2 (see also McKinney 2006; Pu et al. 2015). The flow along large-scale magnetic field lines threading the central black hole is separated into inflow and outflow regions, and is surrounded by the accretion flow and its corona. A global magnetic field and MHD outflow solution is related to the mass loading onto the field lines (Beskin et al. 1998; Beskin \& Nokhrina 2006; Globus \& Levinson 2013; Pu et al. 2015) and is computationally expensive to calculate. For a description mimicking the outflow properties, we instead employ the following working assumptions. We consider a class of stream functions,

$$
\Psi=r^{p}(1-\cos \theta),(0 \leqslant p \leqslant 1),
$$

described in Broderick \& Loeb (2009), which approximately describe the force-free magnetic field (Tchekhovskoy et al. 2008). 


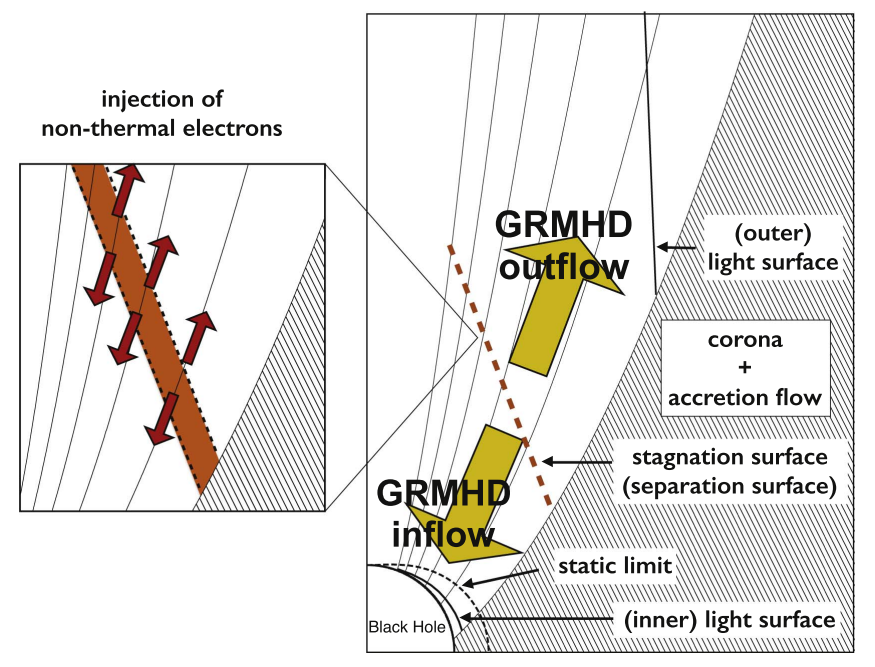

Figure 2. Schematic plot of the GRMHD flow and the black hole magnetosphere. We assume that the non-thermal electrons (red arrows) are generated at the stagnation surface (red region) and consider their subsequent cooling when they are embedded in the background GRMHD inflow and outflow (yellow arrows).

\subsection{Stagnation Surface}

Along a rigidly rotating field line, the stagnation surface can be estimated from the location where the magnetocentrifugal force is balanced by the gravity of the black hole (Takahashi et al. 1990). The stagnation surface can be located by searching for where $K_{0}^{\prime}=0$ along the field line, where $K_{0} \equiv$ $-\left(g_{\phi \phi} \Omega_{\mathrm{F}}^{2}+2 g_{t \phi} \Omega_{\mathrm{F}}+g_{t t}\right)$ and $\Omega_{\mathrm{F}}$ is the angular velocity of the field. Inside (outside) the stagnation surface, the flow is accelerated inward (outward) and becomes an inflow (outflow). For a cold flow within which the pressure may be ignored, the flow begins with zero velocity at the location where $K_{0}^{\prime}=0$ (the acceleration is cancelled out). In a more physically realistic scenario, the velocity of a hot flow at $K_{0}^{\prime}=0$ does not begin with zero velocity. Here we assume that the inflow and outflow solutions (as described below) begin at $K_{0}^{\prime}=0$, and that the non-thermal electrons are generated therein in a stationary manner.

\subsection{Flow Dynamics}

For outflow along the field lines, we also adopt the aforementioned force-free model, describing the fluid motion and magnetic field strength for the region where the field lines thread the black hole event horizon. Although this semianalytic description has the drawback of being a force-free jet model which cannot reflect the intrinsic particle acceleration properties of GRMHD processes (related to the mass loading), it greatly simplifies numerical calculations while still providing a sensible qualitative model for the flow dynamics. We therefore adopt this approach as a starting point in this work, leaving a more in-depth treatment to a future work. Noting that the drift velocity is always positive (outward), we artificially exclude the GRMHD inflow region inside the stagnation surface so as to match the characteristic features of a GRMHD jet.

The dynamics of the inflow region are obtained by solving the equations of motion along a given field line, i.e., the
Table 1

Model Parameters (See also Section 4.1)

\begin{tabular}{lcll}
\hline \hline & $M_{\mathrm{BH}}\left(M_{\odot}\right)$ & \multicolumn{1}{c}{$a$} & $\theta_{\mathrm{h}}$ \\
\hline M9a9h60 (fiducial) & $6 \times 10^{9}$ & 0.998 & $60^{\circ}$ \\
M9a5h60 & $6 \times 10^{9}$ & 0.5 & $60^{\circ}$ \\
M9a9h20 & $6 \times 10^{9}$ & 0.998 & $20^{\circ}$ \\
M6a9h60 & $4 \times 10^{6}$ & 0.998 & $60^{\circ}$ \\
\hline
\end{tabular}

relativistic Bernoulli equation in the cold limit. As shown in $\mathrm{Pu}$ et al. (2015), a magnetically dominated GRMHD inflow solution is insensitive to mass loading, because its Alfvén surface and fast surface are constrained to be close to the inner light surface and the event horizon, respectively. Based on such characteristic features, we have the freedom to choose a representative inflow solution for the four-velocity $u^{\mu}$ of the inflow for a given magnetization sufficiently high to result in a negative energy inflow, from which the black hole rotational energy is extracted outward. To avoid numerical stiffness issues caused by small values of $v$ and correspondingly large values of $\gamma$ for synchrotron processes $\left(d \gamma / d s \propto \dot{\gamma}_{\text {rad }} / v \sim \gamma^{2} / v\right.$; see Equations (5) and (10)), we consider a floor value $v_{\text {flr }}$ for the cold flow solution, mimicking the non-vanishing flow velocity of a hot flow (where the sound speed is not zero) near the stagnation surface. If the flow velocity $\left|u^{r} / u^{t}\right|$ is below the floor value $v_{\mathrm{flr}}$, both synchrotron processes and adiabatic processes are not considered. The magnetic field structure is determined for both the inflow and outflow regions from the force-free model, and its normalization, $B_{0}$, is a free parameter chosen according to the particular physical scenario.

\subsection{Fluid Mass Density}

In both the inflow and outflow regions, as required by continuity, the fluid mass density (mass loading) can be determined from the conserved quantities $\eta(\Psi)$ (particle flux per unit flux tube) along each field line (e.g., Takahashi et al. 1990), which gives the number density of thermal electrons as

$$
n_{\mathrm{th}}=n_{0} \frac{\eta(\Psi) \Psi_{, \theta}}{\sqrt{-g} u^{r}}>0,
$$

where $g$ is the determinant of the Kerr metric, $u^{r}$ is the radial component of the four-velocity, and $n_{0}$ is a normalization for the number of thermal electrons, $n_{\text {th }}$ (c.f. Broderick \& Loeb 2009). Applying $\rho \propto n_{\text {th }}$ to Equation (13), the value of $\eta(\Psi)$ is not relevant when considering $\rho / \rho_{0}$.

\section{Calculations and Results}

\subsection{Model Parameters}

We consider jet models with $p=1$ and $\Omega_{\mathrm{F}}(\Psi)=0.5 \Omega_{\mathrm{BH}}$, where $\Omega_{\mathrm{BH}}$ is the angular velocity of the black hole. The normalization for the magnetic field strength is chosen to give a field strength $\sim 10 \mathrm{G}$ (with a corresponding $B_{0}=50 \mathrm{G}$ ), in order to fit the inferred value for M87 (Dexter et al. 2012). The model parameters are summarized in Table 1 for different black hole masses and spin parameters, and the calculation is performed along a representative field line $\Psi$ that attaches onto the event horizon at $\theta_{\mathrm{h}}$. We present in Figure 3 the poloidal distance $s$ and magnetic field strength for different models. The 

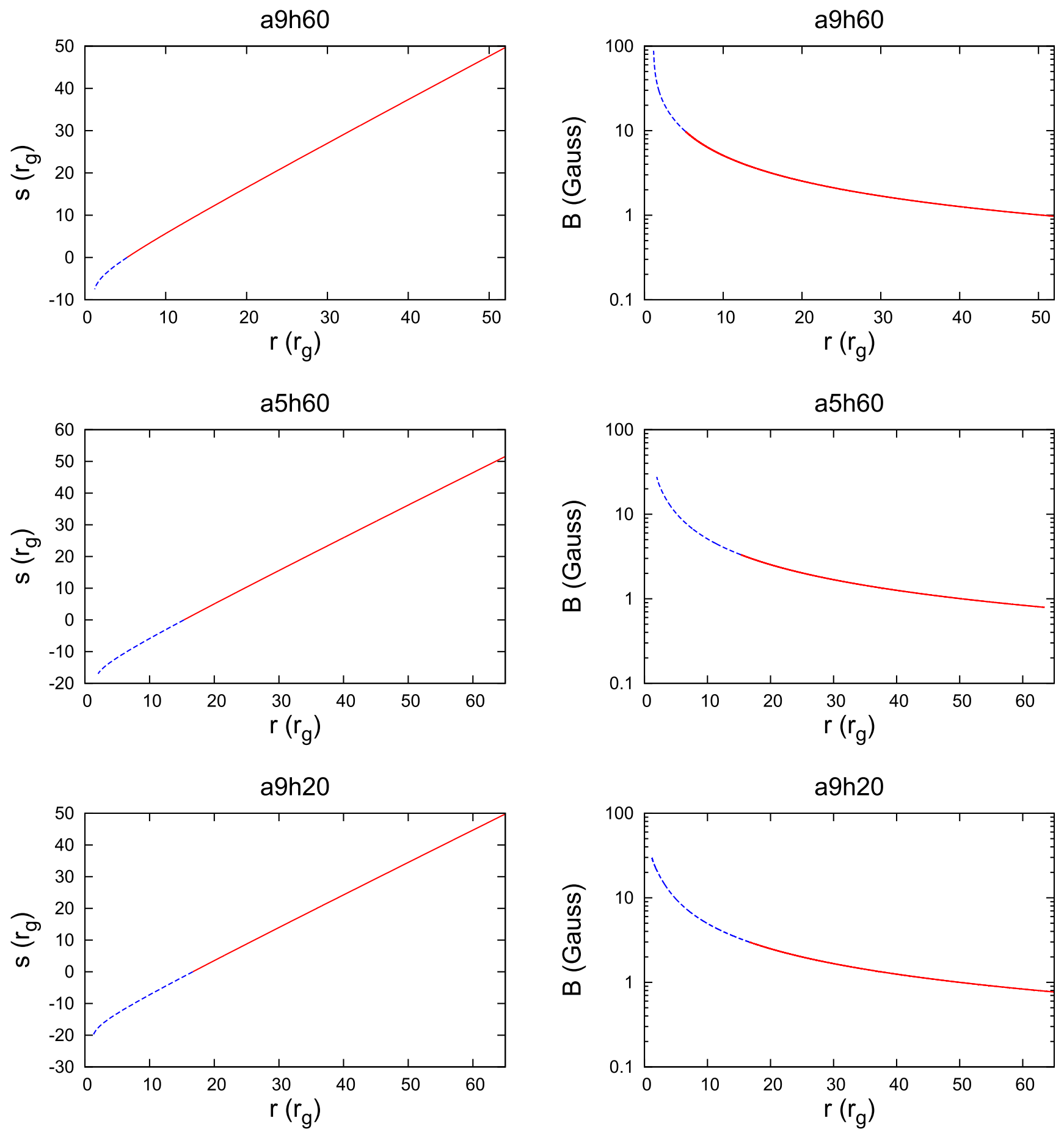

Figure 3. Poloidal distance $s$ and magnetic field strength $B$ (in Gauss) along the field line for each model, in terms of the Boyer-Lindquist radial coordinate, $r$, in units of $r_{\mathrm{g}}$. Inflow and outflow regions are indicated by the blue dashed and red solid segments, respectively. The stagnation surface is located at $s=0$.

GRMHD flow solutions used for each model are presented in the Appendix. Other choices of $p$ and $\Omega_{\mathrm{F}}$ give a qualitatively similar flow structure.

In the fiducial model, M9a9h60, the assumed parameter values are $M_{\mathrm{BH}}=6 \times 10^{9} M_{\odot}$ (e.g., Gebhardt et al. 2011) and a rapid black hole spin of $a=0.998$. The solution is computed along a mid-level field line which attaches onto the event horizon at $\theta_{\mathrm{h}}=60^{\circ}$. In contrast to the fiducial settings, a modest black hole spin of $a=0.5$ is chosen in model M9a5h60, and a different value of $\theta_{\mathrm{h}}=20^{\circ}$ (but with $a=0.998)$ is chosen in model M9a9h20.
In addition, although the jet model is independent of the black hole mass, the radiative cooling depends on the physical length scale and is therefore a function of the black hole mass (Section 2.2). To provide insight into the mass dependence, we consider in model M6a9h60 a smaller black hole mass that is similar to the case of $\mathrm{Sgr} \mathrm{A}^{*}$, i.e., $M_{\mathrm{BH}}=4 \times 10^{6} M_{\odot}$, while keeping the magnetic field the same as in all other cases. ${ }^{7}$ All calculations include both synchrotron and adiabatic process

\footnotetext{
7 Note that in general the magnetic field strength depends on the accretion rate and the black hole mass.
} 
(i.e., $\dot{\gamma}=\dot{\gamma}_{\text {syn }}+\dot{\gamma}_{\text {adi }}$ ) since we are concerned with $\mathrm{mm} / \mathrm{sub}-\mathrm{mm}$ VLBI observational frequencies. ${ }^{8}$

\subsection{Particle Properties of GRMHD Jets}

\subsubsection{Energy Loss of Non-thermal Electrons}

The energy loss of non-thermal electrons can be described by the characteristic curves $d \gamma / d s$ in Equation (5), which are independent of the choice of $\alpha$ (Section 2.1). In Figure 4, we present the spatial variation of the non-thermal electrons of M9a9h60, provided that non-thermal electrons are generated at the stagnation surface (vertical dashed line). A family of selected characteristic curves of electron energies $\gamma_{0}=1,10,10^{2}, 10^{3}$, and $10^{4}$ at the stagnation surface is shown (where the subscript " 0 " denotes the value at the stagnation surface). A floor value of $v_{\text {flr }}=0.03$ is chosen (Section 3.2) so that no numerical stiffness occurs even for the largest values of $\gamma_{0}$ considered in this study. This choice of $v_{\mathrm{flr}}$ does not change the results qualitatively. Imposing a floor results in a flattened profile near the stagnation surface.

For both inflow and outflow regions, the electron energy drops as the electrons stream along the flow and away from the stagnation surface. The synchrotron process, which is $\propto\left(\gamma^{2}-1\right)$, results in a more rapid energy decrease for higher electron energies $\gamma$ (top panel). As the magnetic field strength decreases along the streamline, the effect of synchrotron cooling become less efficient, and the profile of the characteristic curve flattens at larger distances in the outflow region. Adiabatic processes on the other hand, which are $\propto(\gamma-1)$, are insensitive to the electron energy (middle panel). The adiabatic process could result in a heating or cooling process, according to the profile of $\rho$. It is interesting to note that $\rho$ is roughly inversely proportional to $u^{r}$ (Section 3.3), and $u^{r}$ has in general an increasing profile away from the stagnation surface (Figures 10, 11, and 12 in the Appendix). As a result, the profile of $\rho$ is always decreasing away from the stagnation surface, and hence adiabatic cooling takes place in both inflow and outflow regions (see Equation (13)). For the combination of these two processes (bottom panel), profiles of $\gamma$ in the outflow region decay more rapidly compared to when only a single process is considered. For all cases, electrons with $\gamma=1$ do not gain or lose energy, as expected. In the following, we compare the results of other models with those of the fiducial model (M9a9h60).

The electron-synchrotron absorption cross-section is

$$
\sigma_{\text {syn }}(\nu, B, \sin \theta ; \gamma)=\frac{8 \pi^{2}(3 \sin \theta)^{2 / 3} \Gamma(5 / 3)}{3 \sqrt{3}}\left(\frac{e}{B}\right)\left(\frac{\nu_{\mathrm{L}}}{\gamma \nu}\right)^{5 / 3}
$$

(see, e.g., Ghisellini 2013), where $\gamma$ is the Lorentz factor of the non-thermal electrons, which gives the optical depth

$$
\tau_{\text {syn }} \approx 1.09 \times 10^{8}\left(\frac{n_{\mathrm{e}}}{10^{5} \mathrm{~cm}^{-3}}\right)\left(\frac{R}{10^{12} \mathrm{~cm}}\right)\left(\frac{10 \mathrm{G}}{B}\right)\left(\frac{\nu_{\mathrm{L}}}{\gamma \nu}\right)^{5 / 3},
$$

where $n_{\mathrm{e}}$ is the non-thermal electron number density and $R$ is the size of the emission region. For a magnetic field $B=10 \mathrm{G}$, the Larmor frequency $\nu_{\mathrm{L}}$ $(=28 \mathrm{MHz})$ is much lower than the observational frequency $\nu(\sim 100 \mathrm{GHz}$ or higher). With $R=10^{12} \mathrm{~cm}, B=10 \mathrm{G}$, and $\nu=100 \mathrm{GHz}$,

$$
\tau_{\text {syn }} \approx 0.06\left(\frac{n_{\mathrm{e}}}{10^{5} \mathrm{~cm}^{-3}}\right)\left(\frac{10^{2}}{\gamma}\right)^{5 / 3} .
$$

For magnetic aligned flows, $B \sim 10 \mathrm{G}$ implies $n_{\mathrm{e}} \ll \rho / m_{\mathrm{p}} \ll 10^{5} \mathrm{~cm}^{-3}$, where $m_{\mathrm{p}}$ is the proton mass. Hence, synchrotron self-absorption is unimportant in the jets considered in this work.
Figure 5 shows the result of model M9a5h60. Due to the slower black hole spin $(a=0.5)$, the stagnation surface is located farther away from the black hole (see also Figure 3). The location of the stagnation surface farther from the black hole affects the relative importance of synchrotron and adiabatic cooling for electrons with different initial energies. For synchrotron cooling, the electron energy decays less rapidly from the stagnation surface because the magnetic field is weaker, given that the normalizations for the magnetic fields are the same in both models. Due to the energy dependence of synchrotron cooling $\left(\propto \gamma^{2}-1\right)$ and adiabatic cooling $(\propto \gamma-1)$, electrons with higher (or lower) energy more easily lose energy via synchrotron cooling (or adiabatic cooling), for both inflow and outflow regions.

For identical black hole spin parameters, the location of the stagnation surface is farther away from the black hole for field lines at higher latitudes (see also Figure 3). Figure 6 shows the result for model $\mathrm{M} 9 \mathrm{a} 9 \mathrm{~h} 20\left(\theta_{\mathrm{h}}=20^{\circ}\right)$; the case where a field line close to the polar region is considered. Similarly, the farther-removed location of the stagnation surface causes electrons to lose energy less rapidly, and the electrons with lower energy lose their energy via adiabatic cooling more easily due to the weaker synchrotron cooling.

The spatial variation of the electron energy for M6a9h60 $\left(M_{\mathrm{BH}}=4 \times 10^{6} M_{\odot}\right)$, the smaller black hole mass case, is shown in Figure 7. The dynamical timescale of the flow in this case is smaller than in the previous cases where the black hole is 1000 times more massive. The top panel clearly shows that synchrotron loss is insignificant in this case. This can be understood as the synchrotron loss timescale being much longer than the dynamical timescale of the flow. Comparing the middle and the bottom panels confirms that the cooling is dominated by adiabatic processes, of which the adiabatic cooling timescale is governed by the dynamical timescale of the background GRMHD flow considered here.

\subsubsection{Spatial Variation of the Distribution of Non-thermal Electrons}

The non-thermal electron distribution $n_{\text {nth }}(\gamma, s)$ is related to the thermal electron distribution $n_{\text {th }}(s)$ determined by the GRMHD flow and the distribution function $\left.\mathcal{G}(\gamma)\right|_{s}$ (Equation (2)). According to the profile of $\mathcal{F}(\gamma, s)$, the distribution function $\left.\mathcal{G}(\gamma)\right|_{s}$ can be computed at a given slice of constant $s$ (Equation (2)). In the following, we present the spatial evolution of $\left.\mathcal{G}(\gamma)\right|_{s}$ for different injected electron energy distributions (Equation (8)) as $\gamma_{0}^{-\alpha}$, with $\alpha=1.2,2$, and 2.2. In each case, we assume $1 \leqslant \gamma_{0} \leqslant 1000$, and focus on the result when both synchrotron cooling and adiabatic cooling are included. Interestingly, the spatial evolution has different characteristics depending on whether synchrotron or adiabatic processes dominate. Here, we select M9a9h60 and M6a9h60 as representative cases for these two situations.

The spatial variations of $\left.\mathcal{G}(\gamma)\right|_{s}$ at different locations of the inflow and outflow regions of M9a9h60 are shown in Figure 8. Starting from an initial distribution $\gamma_{0}^{-\alpha}$ given at $s_{0}$ (red line), profiles of the slices at the locations of decreasing $s$ (downstream from the stagnation surface) no longer follow a power-law profile. The variations of each slice with decreasing $s$ are indicated by the arrows in each plot. For both the inflow (left panel) and the outflow (right panel), the maximum electron energy $\gamma_{\max }$ at each slice is determined by the characteristic curve with $\gamma_{0}=1000$ as shown in the bottom panel of Figure 4, and is therefore 

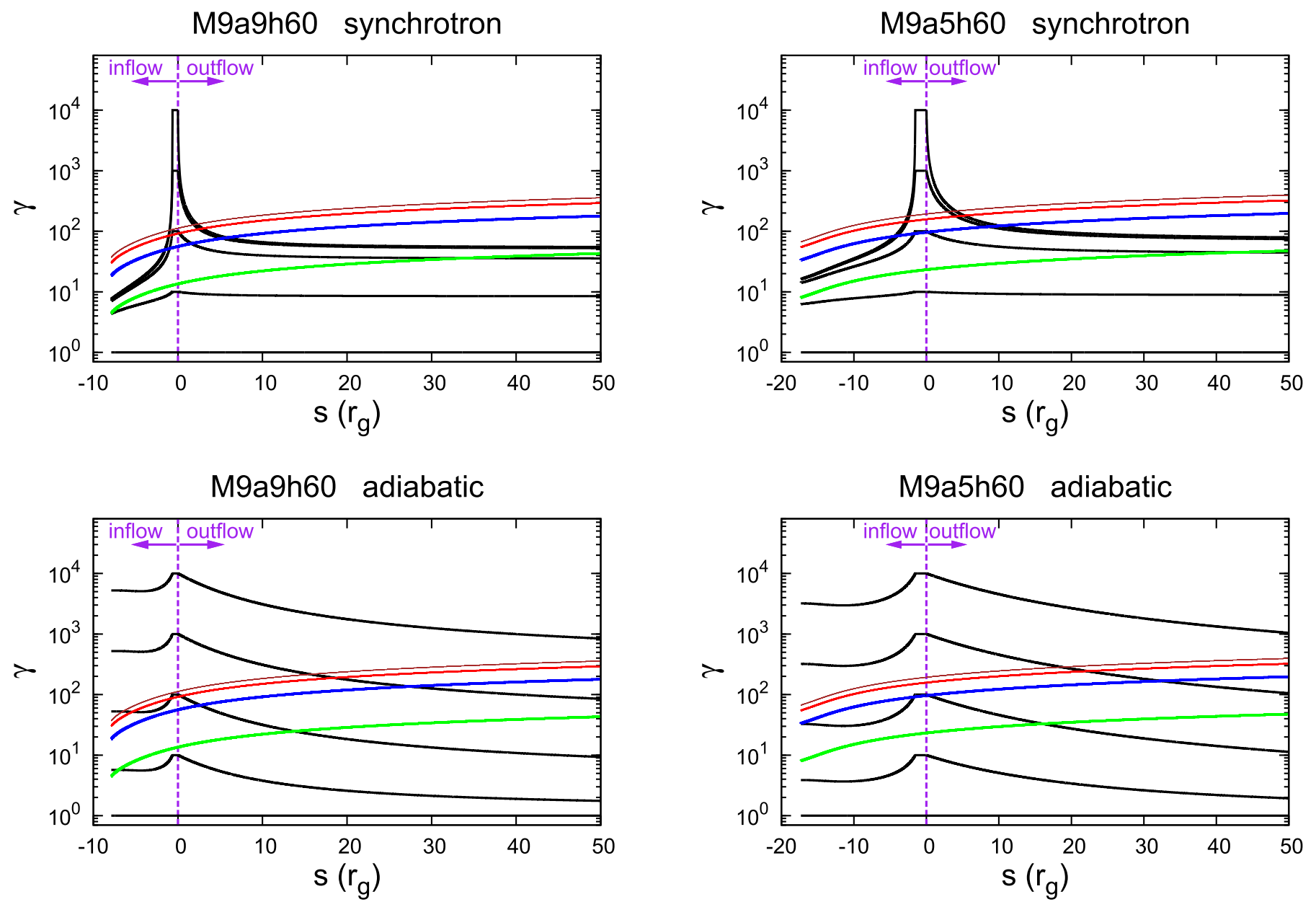

M9a9h60 synchrotron \& adiabatic

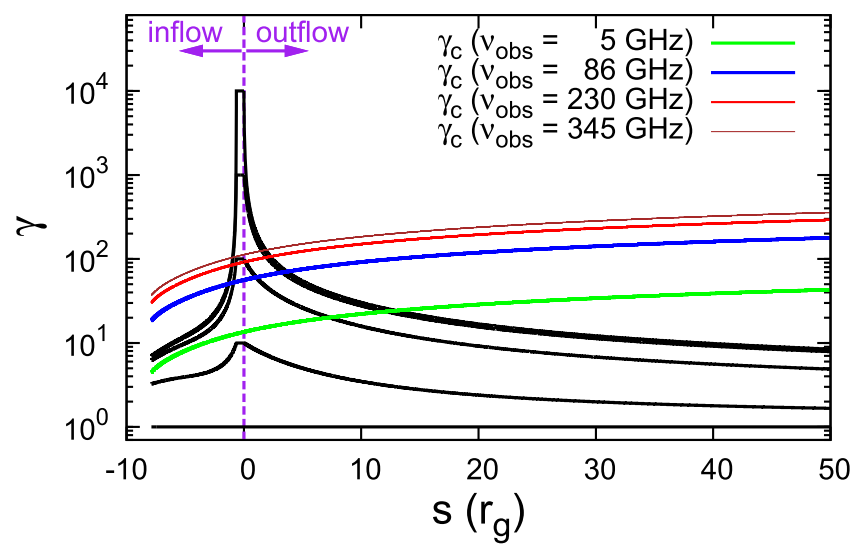

Figure 4. Spatial variations of the non-thermal electron energy along a midlatitude field line for model M9a9h60. Selected characteristic curves (black) from Equation (5) represent injected electrons with initial energies of $\gamma_{0}=1,10,10^{2}, 10^{3}$, and $10^{4}$ at the stagnation surface $(s=0$, as indicated by the vertical dashed line), and their spatial variation as they stream along the background GRMHD inflow region $(s<0)$ and outflow region $(s>0)$. Shown separately are the characteristic curves due to synchrotron processes only (top), adiabatic processes only (middle), and both processes (bottom). The flattened profile to the left of the stagnation surface (Section 3.2) is due to the use of $v_{\text {flr }}$ (see text) and does not qualitatively change these results. The characteristic electron energies $\gamma_{c}$ for producing emission at observational frequencies of $\nu_{\mathrm{obs}}=5 \mathrm{GHz}$ (green), $86 \mathrm{GHz}$ (blue), $230 \mathrm{GHz}$ (red), and $345 \mathrm{GHz}$ (brown) are also overlaid (see Section 4.3). 

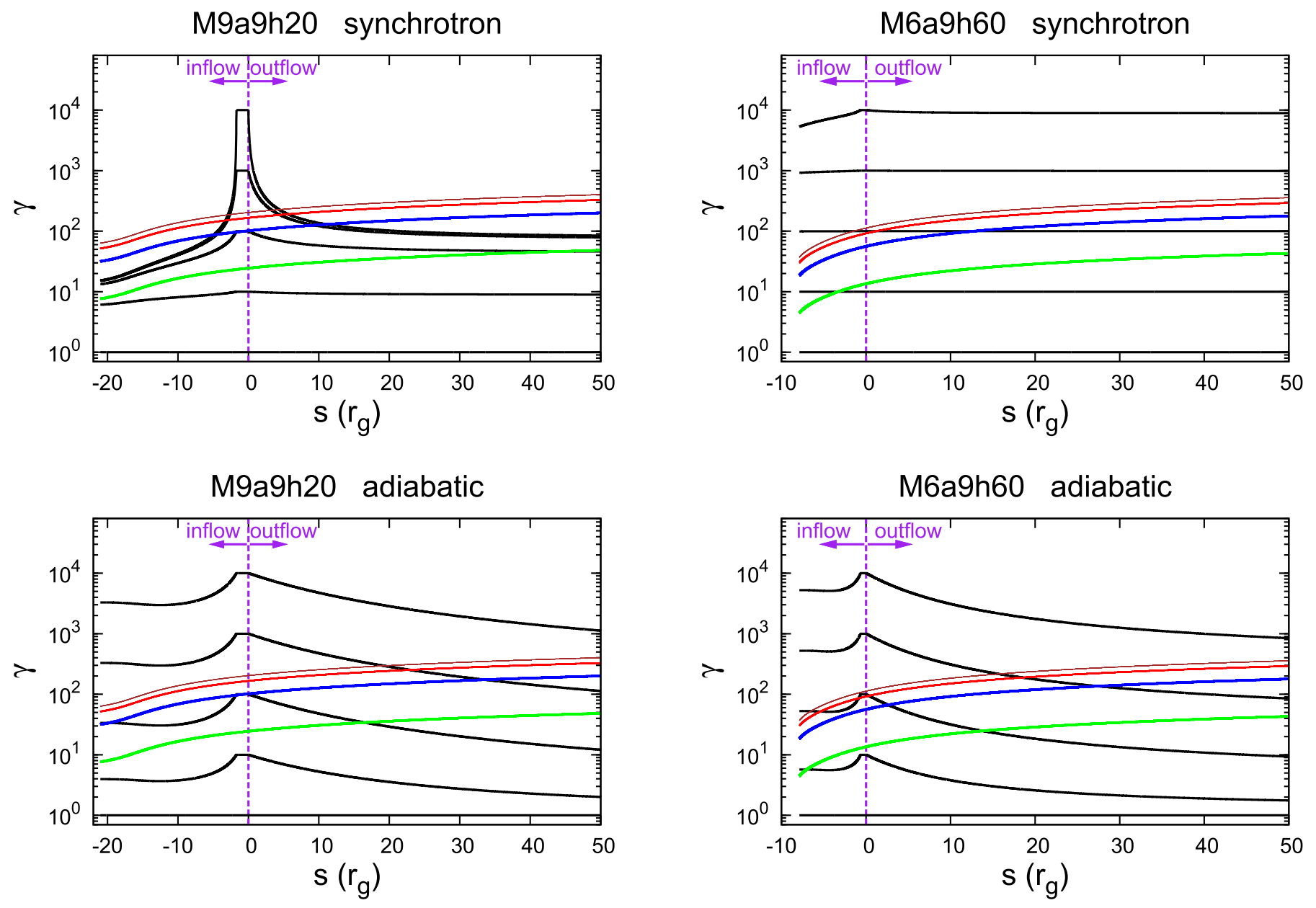

M9a9h20 synchrotron \& adiabatic

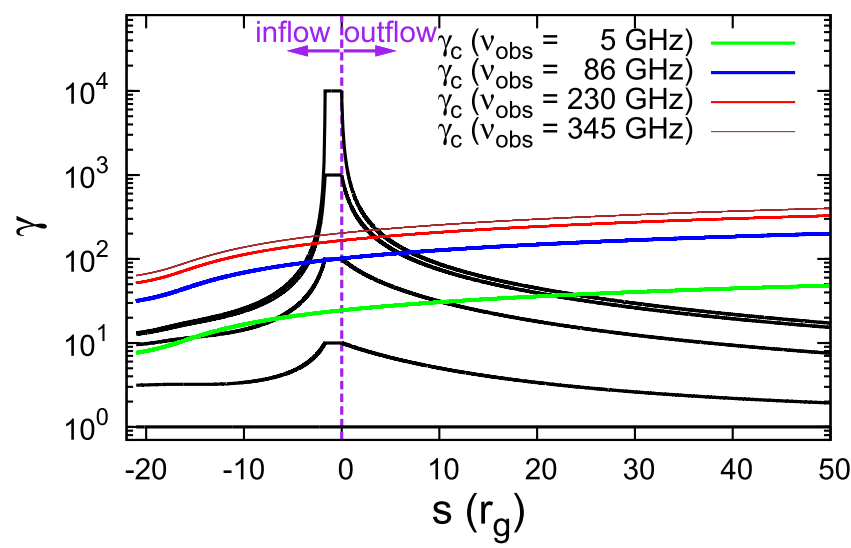

Figure 6. Spatial variations of the non-thermal electron energy along a highlatitude field line for model M9ah20. See Figure 4 for a description and comparison.

$-\alpha=-2$ is preserved. Note also the conservation of the total area of $\mathcal{G}(\gamma)$ at each slice as required by Equation (3). Therefore, a sharp decrease in $\gamma_{\max }$ and a flatter slope results in profiles of $\left.\mathcal{G}(\gamma)\right|_{s}>\left.\mathcal{G}(\gamma)\right|_{s_{0}}$ (top panel).

The distribution function of M6a9h60 shown in Figure 9 provides an example of the case where adiabatic processes dominate $(\propto \gamma-1)$. Similar to the previous discussion, the

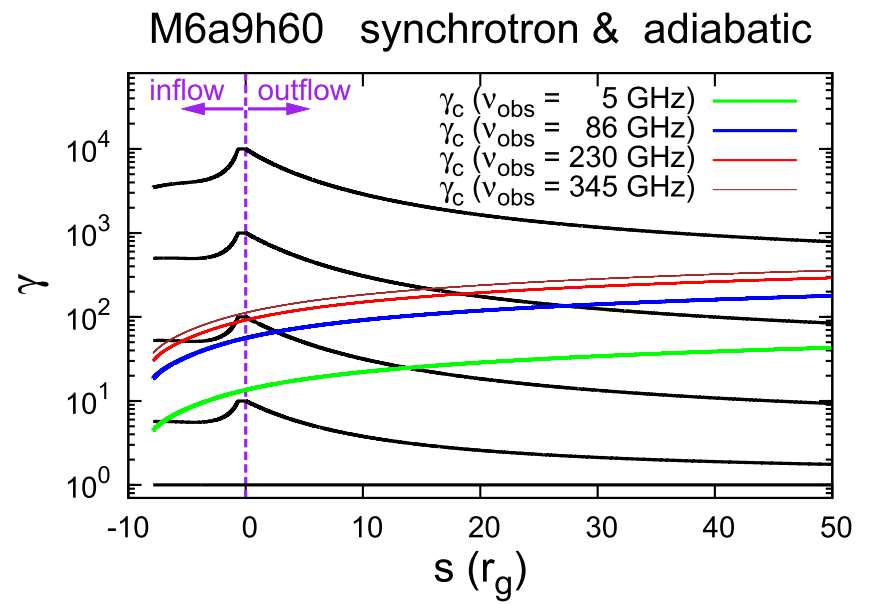

Figure 7. Spatial variations of the non-thermal electron energy along a midlatitude field line for model M6a9h60. See Figure 4 for a description and comparison.

profile of $\gamma_{0}=1000$ in Figure 7 provides a reference for the maximum value of $\gamma$ on the same slices. Due to cooling processes, the fraction of electrons with $\gamma \rightarrow 1$ increases. There are two striking differences when compared to the synchrotron process-dominated case. First, for all choices of $\alpha$, $d(\ln \mathcal{G}) / d(\ln \gamma)$ is always negative at the high-energy end. The slope $-\alpha$ is preserved at different spatial locations when 

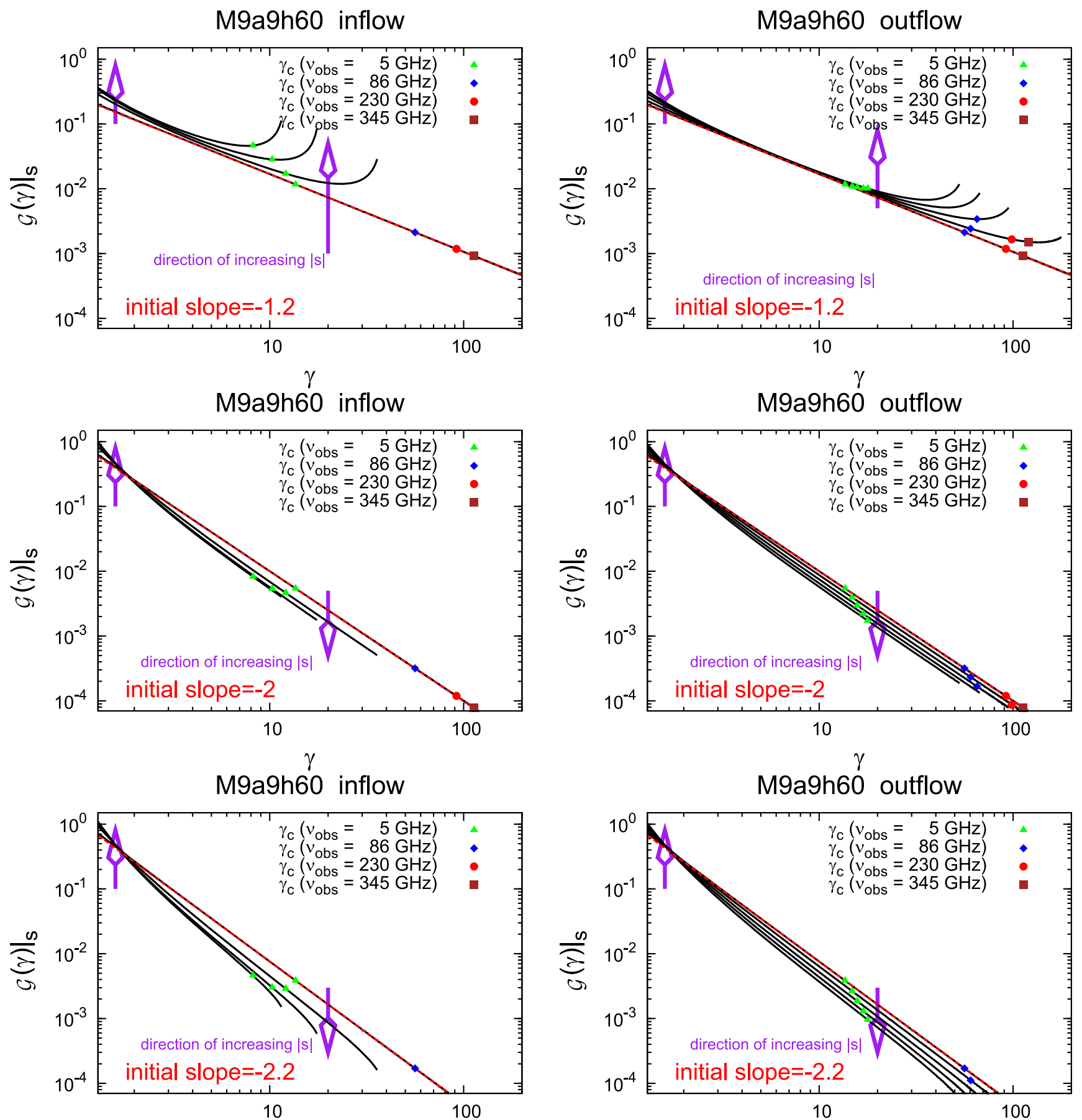

Figure 8. Spatial variation of the distribution function $\mathcal{G}(\gamma)$ of model M9a9h60 at different locations along the inflow (left panel) and outflow (right panel). The profiles are of $\left.\mathcal{G}(\gamma)\right|_{s}$, with slices chosen to have a fixed interval of $r$ along the line. For the inflow, $r=r_{0}, 4,3$, and $2 r_{\mathrm{g}}$ are chosen, where $r_{0}=\left.r\right|_{s_{0}} \simeq 5.2 r_{\mathrm{g}}$ is the $r$ coordinate at the stagnation surface $s_{0}$. For the outflow, $r=r_{0}, 6,7,8$, and $9 r_{\mathrm{g}}$ are chosen. At the stagnation surface, the prescribed initial distribution follows $\left.\mathcal{G}(\gamma)\right|_{s_{0}} \propto \gamma_{0}^{-\alpha}$ and $1 \leqslant \gamma_{0} \leqslant 10^{3}$, with $\alpha=1.2$ (top panels), 2.0 (middle panels), and 2.2 (bottom panels). The initial distribution at $r_{0}$ overlaps with the red dashed line, and the subsequent thread of the evolution is indicated by the arrows. At each slice of constant $r$, electron energies $\gamma$ that are equal to the corresponding $\gamma_{\mathrm{c}}$ for observational frequencies of $\nu_{\mathrm{obs}}=5 \mathrm{GHz}$ (green), $86 \mathrm{GHz}$ (blue), $230 \mathrm{GHz}$ (red), and $340 \mathrm{GHz}$ (brown) are indicated by the colored symbols described in the panel legend.

adiabatic processes dominate (Equation (21)). Second, the maximum electron energy does not change as rapidly as in the case of synchrotron processes $\left(\propto \gamma^{2}-1\right)$.

\subsection{Implications}

For non-thermal electrons, most synchrotron emission may be attributed to electrons with characteristic energy $\gamma_{\mathrm{c}}^{2} \sim \nu_{\mathrm{obs}} / \nu_{\mathrm{c}}$ (e.g., Rybicki \& Lightman 1986; Leung et al.
2011), where $\nu_{\mathrm{obs}}$ is the observed frequency and $\nu_{\mathrm{c}} \equiv e B /\left(2 \pi m_{\mathrm{e}} c\right)$. After some computation, we obtain

$$
\gamma_{\mathrm{c}} \sim 59.8 \sqrt{\left(\frac{\nu_{\mathrm{obs}}}{100 \mathrm{GHz}}\right)\left(\frac{10 \mathrm{G}}{B}\right)} .
$$

For our frequencies of interest, $\nu_{\mathrm{obs}}=5,86,230$, and $345 \mathrm{GHz}$, the corresponding profiles of $\gamma_{\mathrm{c}}\left(\nu_{\mathrm{obs}}\right)$ along the field 

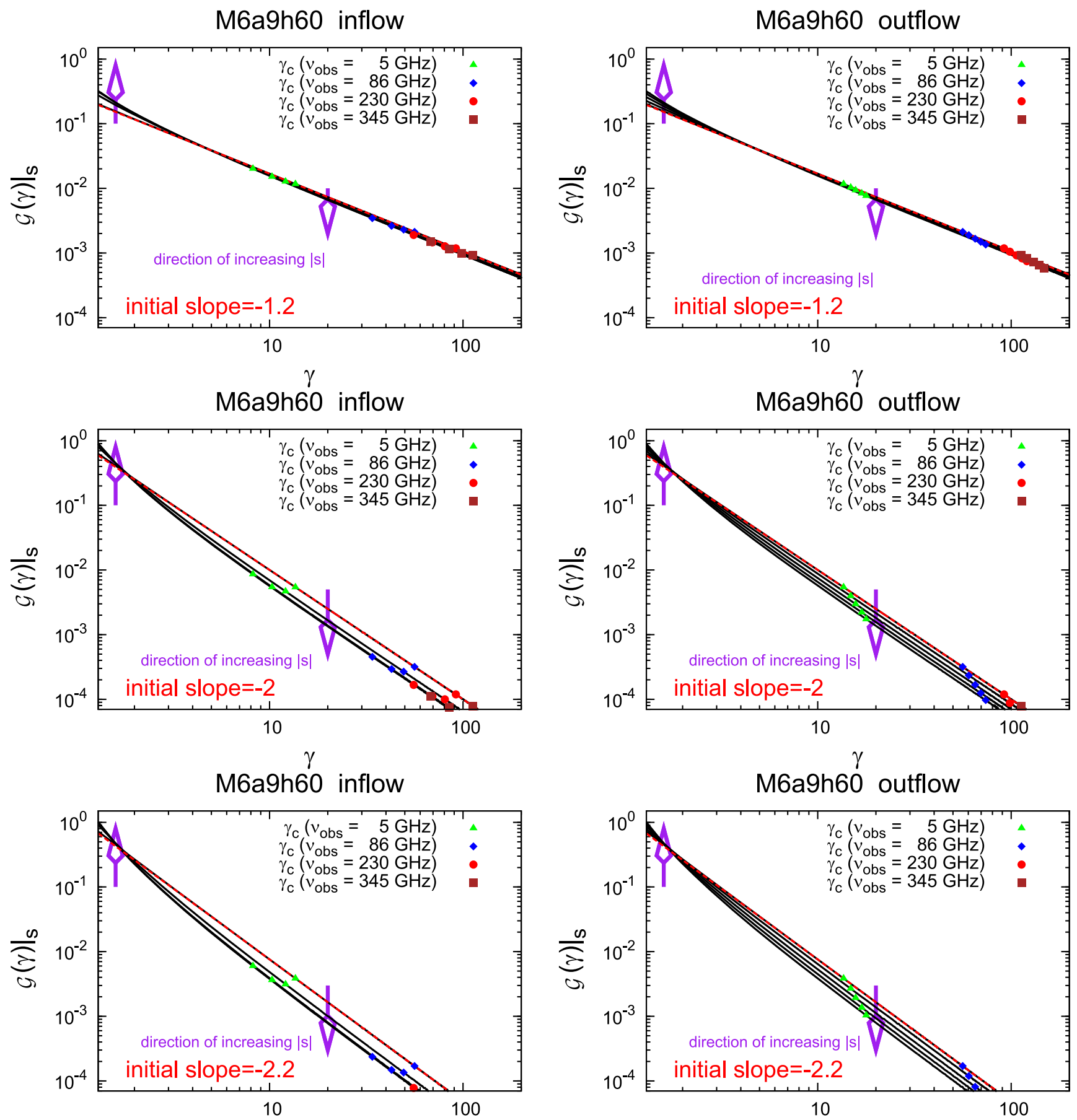

Figure 9. Spatial variation of the distribution function $\mathcal{G}(\gamma)$ of model M5a9h60 at different locations along the inflow (left panel) and outflow (right panel). See the caption of Figure 8 for further details.

line for each model overlap in Figures 4-7. With the magnetic field strength decreasing farther away from the black hole, $\gamma_{\mathrm{c}}$ is monotonically increasing along the field line. The nature of the decreasing profile of characteristic curves and the increasing profile of $\gamma_{c}$ indicates a finite non-thermal synchrotron emission region within which

$$
\gamma_{\max }(s ; \Psi)>\gamma_{\mathrm{c}}(s ; \Psi)
$$

is satisfied. It is clear that the size of the emitting region therefore depends on the observational frequency $\gamma_{\mathrm{c}}\left(\nu_{\mathrm{obs}}\right)$ and the variation of electron energy along a given field line $\gamma_{\max }(s ; \Psi)$. As shown in Figures 4-7, the emission region size becomes larger when the observational frequency decreases, with the stagnation surface being farther away, or the flow being dominated by adiabatic processes. As the observational frequency increases, the emission region size decreases and gradually coincides with the birth place of non-thermal 
electrons. That is to say, the location of the stagnation surface becomes increasingly apparent as the observational frequency increases. For a given field configuration $\Psi$, the location of the stagnation surface is related to the black hole spin parameter and the angular velocity of the field $\Omega_{\mathrm{F}}$ (Section 3.1). As a result, it may be possible to constrain the spin of the black hole using the information concerning the location of the stagnation surface, as provided by VLBI observations at multiple frequencies. Observations of non-thermal synchrotron emission of the M87 jet beyond parsec scales (e.g., Perlman et al. 1999, 2001; Homan \& Lister 2006; Hada et al. 2011; Mertens et al. 2016) indicate that subsequent reheating (injecting energy into electrons) far from the emission region discussed here is necessary.

The shape of the emitting region can be inferred as a collection of non-thermal electron distributions on different field lines. Comparing the results of M9a9h20 $\left(\theta_{\mathrm{h}}=20^{\circ}\right)$ and those of M9a9h60 $\left(\theta_{\mathrm{h}}=60^{\circ}\right)$ for higher latitude field lines, the stagnation surface is located farther away from the black hole and the emission region size appears larger (relatively) along the field line. It is expected that the emitting region is more extended toward the jet axial region.

In Figures 8 and 9, we indicate the electrons with $\gamma$ equal to the corresponding $\gamma_{c}$ for different observational frequencies at each slice of fixed spatial location. If $\gamma_{c}$ exceeds $\gamma_{\max }$, the ensemble of electrons at a specific slice can no longer contribute emission at any of the given observational frequencies, and no indicated $\gamma_{c}$ appears on that slice. It is expected that the emission close to the stagnation surface would be exceptionally luminous due to the larger electron number density there. For the outflow, such a condition will eventually be satisfied due to both synchrotron cooling and adiabatic cooling (left panels of Figures 8 and 9).

For the inflow, whether or not the electrons can contribute to the synchrotron emission (i.e., if $\gamma_{\max }(s ; \Psi)>\gamma_{\mathrm{c}}(s ; \Psi)$ is satisfied) all the way down to the event horizon depends on how quickly the non-thermal electrons lose their energy. If synchrotron cooling is efficient (which is the case for a larger black hole mass), the inflow component is only partially observable (as shown by the absence of $\gamma_{c}$ on the slices in the left panels of Figure 8; see also Figure 4). If adiabatic cooling is more important (which is the case for a smaller black hole mass), the whole inflow component may be observable (as shown by the presence of $\gamma_{c}$ on the slices in the left panels of Figure 9; see also Figure 7) since $\gamma_{\max }$ decays at a slower rate $(\propto \gamma-1)$ compared to the synchrotron cooling $\left(\propto \gamma^{2}-1\right)$.

Although the location of the stagnation surface is time dependent, as shown in GRMHD simulations (Broderick \& Tchekhovskoy 2015), a stationarity assumption may be adopted for M87 because of its long dynamical timescale $\left(G M_{\mathrm{BH}} / c^{3} \sim 8 \mathrm{hr}\right)$ compared to typical VLBI observations. Future VLBI observations will provide an excellent opportunity to constrain the injection site of non-thermal electrons and test the predictions of different jet origin models. For further comparison, general-relativistic radiative transfer should be included to take into account the energy shift and aberrations due to the fluid motion and the black hole's strong gravitational field (e.g., Fuerst \& Wu 2004, 2007; Schnittman \& Bertschinger 2004; Dexter \& Agol 2009; Vincent et al. 2011; Younsi et al. 2012; Chan et al. 2013; Pu et al. 2016).

\section{Summary and Conclusion}

We determine the energy spectral evolution of non-thermal electrons in a GRMHD black hole-powered jet and calculate the radio synchrotron radiation from these electrons in the jet inflow and outflow regions near the event horizon. Energetic electrons are injected at the stagnation surface, a unique feature predicted by the GRMHD model, and these electrons (Section 2) are subsequently carried by the GRMHD flow along the magnetic field lines threading the black hole event horizon (Section 3). The energy spectra variation of the nonthermal electrons along the flows is regulated by synchrotron radiative losses and adiabatic processes.

More specifically, we assume there is no further injection of non-thermal electrons after they are injected from the stagnation surface with a power-law energy spectrum (Equation (8)), and the scattering and drift along magnetic field lines are unimportant. Without enough time to reach thermalized or equal-partition state, the non-thermal electron distribution $n_{\text {nth }}(\gamma, s)$ is described by Equation (1). At each location $s$, the spatial variation of non-thermal electrons are traced by using the normalized energy spectral distribution function $\left.\mathcal{G}(\gamma)\right|_{s}$ via the presented conservative formula (Section 2.1). The spatial variation of non-thermal electrons energy, $d \gamma / d s$, can therefore be described by the characteristic curves, Equation (5). We summarize our findings in the following five paragraphs.

1. In the outflow region, the energy of the electrons drops due to radiative losses (via synchrotron radiation, $\dot{\gamma} \propto \gamma^{2}-1$ ) and mechanical cooling (via adiabatic expansion of the jet fluid, $\dot{\gamma} \propto \gamma-1$ ). Farther away from the stagnation surface along the magnetic field lines, the energy of the non-thermal electrons drops significantly, so much so that their contribution to the observable synchrotron radiation becomes insignificant. For imaging observations at a fixed frequency band, the emitting region, if it can be resolved, would appear finite in size. The size of the emitting region, however, varies with observational frequency: the smaller the size of the emitting region, the higher the corresponding observational frequency. Moreover, adiabatic expansion is the dominant cooling process for the jet outflow far away from the stagnation surface.

2. In the inflow region, similar to the outflow region, the energy of electrons drops due to both synchrotron cooling and adiabatic cooling. If radiative cooling dominates, the electrons can lose energy rapidly and become negligible in their contribution to the observable emission (at a given observational frequency) before reaching the event horizon. If adiabatic cooling dominates, electrons lose energy less rapidly. As a result, electrons can remain energetic enough to contribute to the observable emission when they approach the black hole event horizon, rendering the entire inflow region observable (until the dimming effect due to gravitational redshift dominates).

3. Our calculations have shown that the relative importance of radiative losses and adiabatic losses is dependent on the dynamical timescale of the flow and hence the black hole mass of the system, provided that a similar magnetic field strength is applied (see Equation (14)). If so, 
synchrotron losses are expected to be the dominant cooling process for systems with a more massive black hole, whereas adiabatic losses would be important for the systems with a smaller mass black hole. As such, M87like and $\mathrm{Sgr} \mathrm{A}^{*}$-like systems could exhibit different observational properties, even when their jets are driven by the same GRMHD process.

4. In studies of emission from relativistic jets in the compact core region, the energy spectra of non-thermal electron populations are often assumed to have a power-law profile with a maximum energy $\gamma_{\max }$. We have shown that, when the energy loss is dominated by synchrotron cooling instead of adiabatic cooling, the non-thermal electrons, which had an initial power-law energy distribution $\gamma_{0}^{-\alpha}$ when they were injected, cannot maintain a power-law spectrum. Furthermore, $\gamma_{\max }$ drops rapidly and the spatial variation of the electron energy density, in terms of $n_{\text {nth }}(\gamma, s)$, depends on the value of the initial spectral index $\alpha$. On the other hand, if the energy loss is dominated by adiabatic cooling, $\gamma_{\max }$ decays less rapidly and the initial power-law energy distribution can be preserved at the higher energy end.

5. The location of the stagnation surface is farther away from the black hole toward the jet central axis, and the radio emission (as observed at a specific frequency) will appear more extended accordingly. As the observational frequency increases, the emitting region will eventually coincide with the stagnation surface, where the nonthermal electrons are freshly injected. The high concentration of fresh energetic electrons near the stagnation surface implies that regions adjacent to the stagnation surface should be luminous and more easily observable.

Our calculations showed strong radio emission from the regions near the stagnation surface. Predicted observed images of this stagnation surface will be explored with generalrelativistic radiative transfer calculations in a future work. The detection of such bright emission features in VLBI imaging observations will unambiguously confirm the GRMHD nature of black hole-powered jets. This is achievable with upcoming $\mathrm{mm} /$ sub-mm VLBI observations.

H.-Y.P., K.A., and M.N. are grateful to the GLT team in ASIAA for their support and encouragement. H.-Y.P. and K.A. are supported by the Ministry of Science and Technology (MOST) of Taiwan, under the grant MOST 103-2112-M-001038-MY2. Z.Y. is supported by an Alexander von Humboldt Fellowship. Z.Y. and Y.M. acknowledge support from the ERC synergy grant "BlackHoleCam: Imaging the Event Horizon of Black Holes" (Grant No. 610058). This research has made use of NASA's Astrophysics Data System.

\section{Appendix \\ Solutions of Background GRMHD Flows}

The GRMHD solutions used for the calculation of the electron energy losses for models M9a9h60 and M6a9h60, model M9a5h60, and model M9a9h20 are presented in Figures 10, 11, and 12, respectively. 

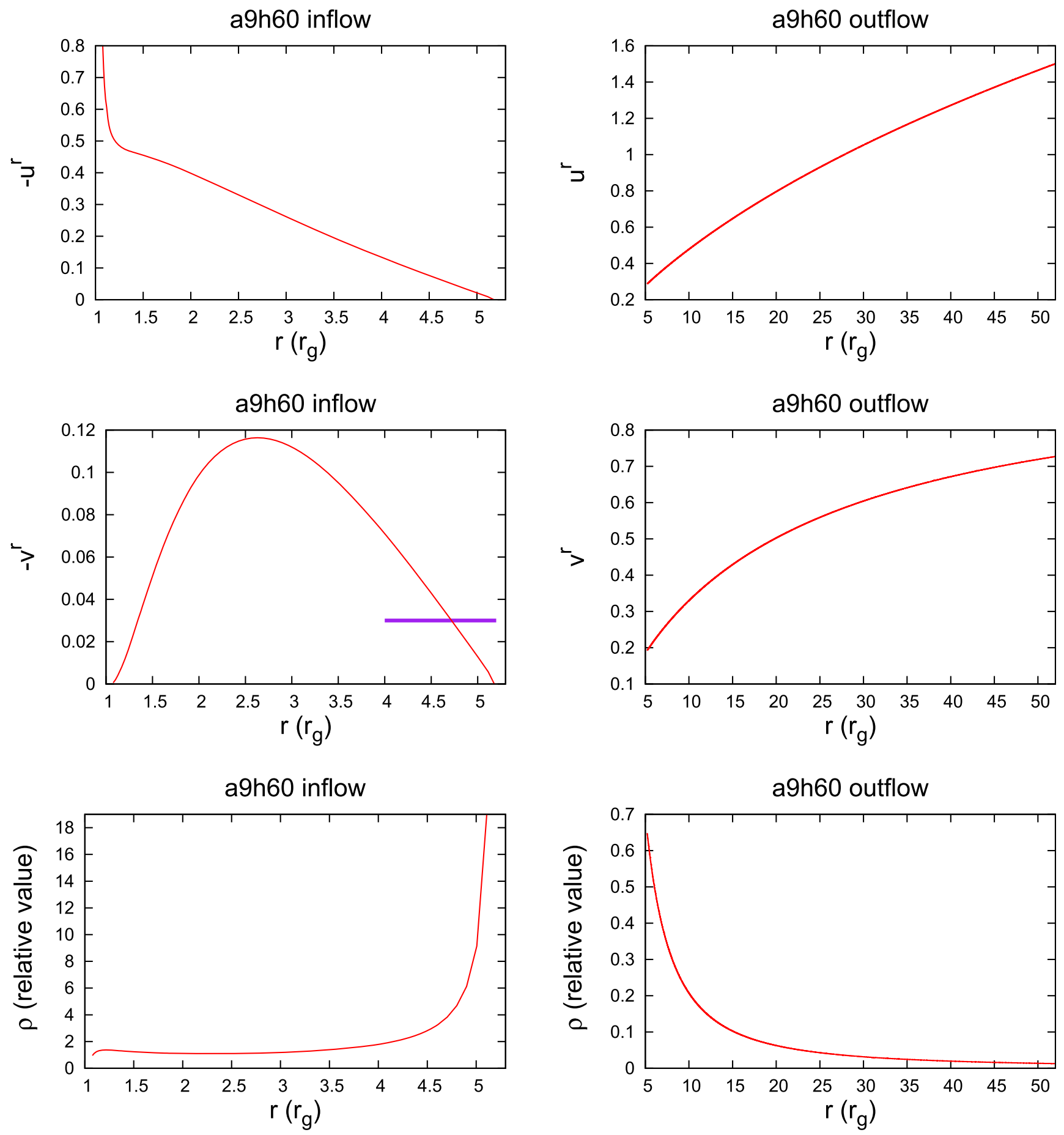

Figure 10. GRMHD solutions for the inflow region (left panels) and the outflow region (right panels) used for models M9a9h60 and M6a9h60, respectively. The profiles are plotted along the field line, in terms of the Boyer-Lindquist radial coordinate $r$. From top to bottom: the radial component of the four-velocity $u^{r}$, the radial component of the three-velocity $v^{r}=u^{r} / u^{t}$ (the applied floor value is indicated by the horizontal line), and the number density $\rho$ (relative numerical value). See Sections 3 and 4.1 for a description and comparison. 

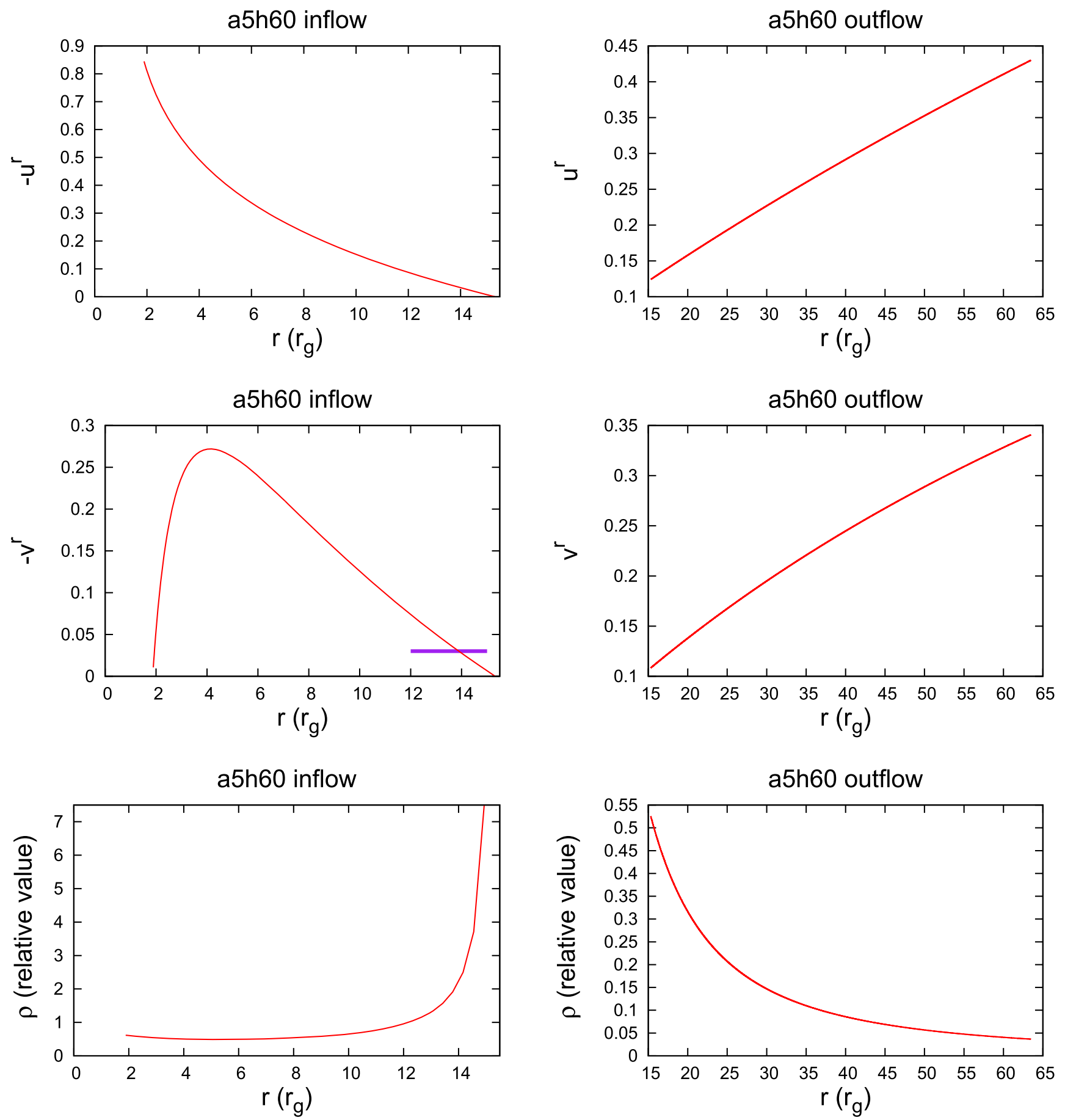

Figure 11. GRMHD solutions for the inflow region (left panel) and outflow region (right panel) used for model M9a5h60. See the caption of Figure 10 for a description and comparison. 

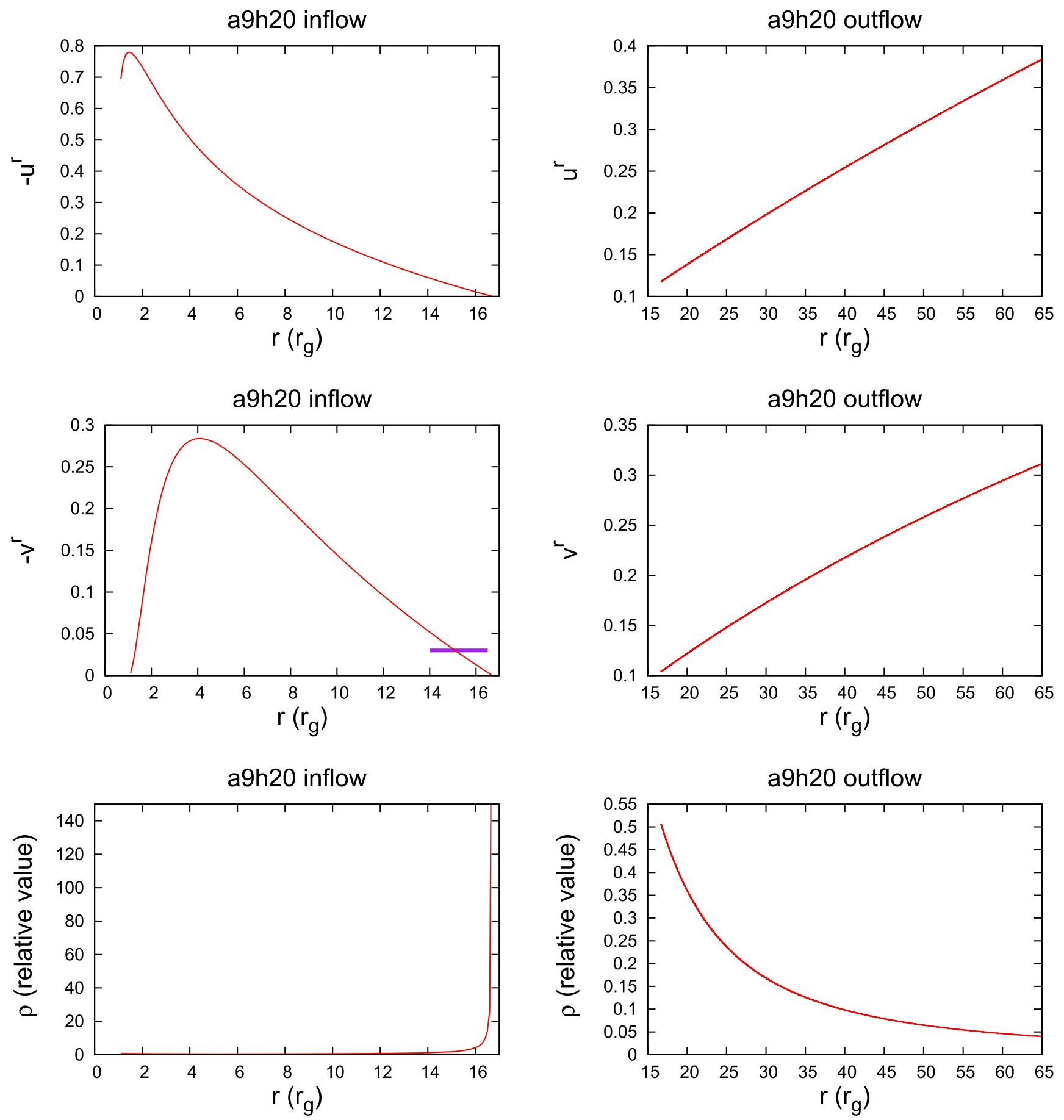

Figure 12. GRMHD solutions for the inflow region (left panel) and outflow region (right panel) used for model M9a9h20. See the caption of Figure 10 for a description and comparison.

\section{ORCID iDs}

Yosuke Mizuno (1) https://orcid.org/0000-0002-8131-6730 Masanori Nakamura (i) https://orcid.org/0000-0001$6081-2420$

\section{References}

Asada, K., Nakamura, M., \& Pu, H.-Y. 2016, ApJ, 833, 56 Bell, A. R. 1978, MNRAS, 182, 147

Beskin, V. S., Kuznetsova, I. V., \& Rafikov, R. R. 1998, MNRAS, 299, 341
Beskin, V. S., \& Nokhrina, E. E. 2006, MNRAS, 367, 375

Blandford, R., \& Eichler, D. 1987, PhR, 154, 1

Blandford, R. D. 1976, MNRAS, 176, 465

Blandford, R. D., \& Königl, A. 1979, ApJ, 232, 34

Blandford, R. D., \& Znajek, R. L. 1977, MNRAS, 179, 433

Broderick, A. E., \& Loeb, A. 2009, ApJ, 697, 1164

Broderick, A. E., \& McKinney, J. C. 2010, ApJ, 725, 750

Broderick, A. E., \& Tchekhovskoy, A. 2015, ApJ, 809, 97

Camenzind, M. 1986a, A\&A, 156, 137

Camenzind, M. 1986b, A\&A, 162, 32

Camenzind, M. 1987, A\&A, 184, 341

Chael, A., Narayan, R., \& Sadowski, A. 2017, arXiv:1704.05092

Chan, C.-K., Psaltis, D., \& Özel, F. 2013, ApJ, 777, 13 
Clausen-Brown, E., Lyutikov, M., \& Kharb, P. 2011, MNRAS, 415, 2081

Dexter, J., \& Agol, E. 2009, ApJ, 696, 1616

Dexter, J., McKinney, J. C., \& Agol, E. 2012, MNRAS, 421, 1517

Doeleman, S. S., Fish, V. L., Schenck, D. E., et al. 2012, Sci, 338, 355

Fender, R. P., Belloni, T. M., \& Gallo, E. 2004, MNRAS, 355, 1105

Fendt, C., \& Greiner, J. 2001, A\&A, 369, 308

Ferreira, J., Petrucci, P.-O., Henri, G., Saugé, L., \& Pelletier, G. 2006, A\&A, 447,813

Fuerst, S. V., \& Wu, K. 2004, A\&A, 424, 733

Fuerst, S. V., \& Wu, K. 2007, A\&A, 474, 55

Gebhardt, K., Adams, J., Richstone, D., et al. 2011, ApJ, 729, 119

Ghisellini, G. 2013, Radiative Processes in High Energy Astrophysics (Berlin: Springer) arXiv:1202.5949

Ginzburg, V. L., \& Syrovatskii, S. I. 1964, SvA, 8, 342

Globus, N., \& Levinson, A. 2013, PhRvD, 88, 084046

Hada, K., Doi, A., Kino, M., et al. 2011, Natur, 477, 185

Hawley, J. F., \& Krolik, J. H. 2006, ApJ, 641, 103

Hirotani, K., Pu, H.-Y., Chun-Che Lin, L., et al. 2016, arXiv:1610.07819

Homan, D. C., \& Lister, M. L. 2006, AJ, 131, 1262

Homan, D. C., Lister, M. L., Kovalev, Y. Y., et al. 2015, ApJ, 798, 134

Honda, M. 2010, MNRAS, 409, 1120

Hovatta, T., Aller, M. F., Aller, H. D., et al. 2014, AJ, 147, 143

Inoue, M., Algaba-Marcos, J. C., Asada, K., et al. 2014, RaSc, 49, 564

Ishibashi, W., Auger, M. W., Zhang, D., \& Fabian, A. C. 2014, MNRAS, 443, 1339

Ito, H., Nagataki, S., Ono, M., et al. 2013, ApJ, 777, 62

Khabibullin, I., Medvedev, P., \& Sazonov, S. 2016, MNRAS, 455, 1414

Kino, M., Takahara, F., Hada, K., et al. 2015, ApJ, 803, 30

Koay, J. Y., Vestergaard, M., Bignall, H. E., Reynolds, C., \& Peterson, B. M. 2016, MNRAS, 460, 304

Koide, S., Shibata, K., Kudoh, T., \& Meier, D. L. 2002, Sci, 295, 1688

Komissarov, S. S. 2004, MNRAS, 350, 427

Komissarov, S. S. 2005, MNRAS, 359, 801

Komissarov, S. S., Barkov, M. V., Vlahakis, N., \& Königl, A. 2007, MNRAS, 380,51

Laing, R. A., \& Bridle, A. H. 2002, MNRAS, 336, 328

Leung, P. K., Gammie, C. F., \& Noble, S. C. 2011, ApJ, 737, 21

Levinson, A., \& Rieger, F. 2011, ApJ, 730, 123

Lu, R.-S., Broderick, A. E., Baron, F., et al. 2014, ApJ, 788, 120

Lyubarsky, Y. 2009, ApJ, 698, 1570

Marscher, A. P. 1980, ApJ, 235, 386

Mason, R. E., Ramos Almeida, C., Levenson, N. A., Nemmen, R., \& Alonso-Herrero, A. 2013, ApJ, 777, 164

McKinney, J. C. 2006, MNRAS, 368, 1561

McKinney, J. C., \& Gammie, C. F. 2004, ApJ, 611, 977

McKinney, J. C., \& Narayan, R. 2007, MNRAS, 375, 531

Meier, D. L. 2005, Ap\&SS, 300, 55
Mertens, F., Lobanov, A. P., Walker, R. C., \& Hardee, P. E. 2016, A\&A, 595, A54

Mościbrodzka, M., Gammie, C. F., Dolence, J. C., \& Shiokawa, H. 2011, ApJ, 735,9

Nakamura, M., Algaba, J. C., Asada, K., et al. 2013, European Physical Journal Web of Conferences, 61, 01008

Nakamura, M., \& Asada, K. 2013, ApJ, 775, 118

Pacholczyk, A. G. 1970, Series of Books in Astronomy and Astrophysics (San Francisco: Freeman)

Perlman, E. S., Biretta, J. A., Zhou, F., Sparks, W. B., \& Macchetto, F. D. 1999, AJ, 117, 2185

Perlman, E. S., Sparks, W. B., Radomski, J., et al. 2001, ApJL, 561, L51

Phinney, E. S. 1983, PhD thesis, Univ. Cambridge

Potter, W. J., \& Cotter, G. 2013, MNRAS, 436, 304

Prieto, M. A., Fernández-Ontiveros, J. A., Markoff, S., Espada, D., \& González-Martín, O. 2016, MNRAS, 457, 3801

Pu, H.-Y., Hirotani, K., \& Chang, H.-K. 2012, ApJ, 758, 113

Pu, H.-Y., Nakamura, M., Hirotani, K., et al. 2015, ApJ, 801, 56

Pu, H.-Y., Yun, K., Younsi, Z., \& Yoon, S.-J. 2016, ApJ, 820, 105

Raffin, P., Ho, P. T. P., Asada, K., et al. 2016, Proc. SPIE, 9906 99060U

Ricarte, A., \& Dexter, J. 2015, MNRAS, 446, 1973

Rybicki, G. B., \& Lightman, A. P. 1986, in Radiative Processes in Astrophysics, ed. G. B. Rybicki \& A. P. Lightman (New York: Wiley), 400

Sbarrato, T., Padovani, P., \& Ghisellini, G. 2014, MNRAS, 445, 81

Schnittman, J. D., \& Bertschinger, E. 2004, ApJ, 606, 1098

Scott, A. E., \& Stewart, G. C. 2014, MNRAS, 438, 2253

Shih, H.-Y., \& Stockton, A. 2014, ApJ, 794, 117

Sironi, L., \& Spitkovsky, A. 2011, ApJ, 726, 75

Sironi, L., \& Spitkovsky, A. 2014, ApJL, 783, L21

Takahashi, M., Nitta, S., Tatematsu, Y., \& Tomimatsu, A. 1990, ApJ, 363, 206

Tchekhovskoy, A., McKinney, J. C., \& Narayan, R. 2008, MNRAS, 388 551

Toma, K., \& Takahara, F. 2014, MNRAS, 442, 2855

Trump, J. R., Impey, C. D., Kelly, B. C., et al. 2011, ApJ, 733, 60

Tucker, W. 1975, Radiation Processes in Astrophysics (Cambridge, MA: MIT Press), 320

Turner, R. J., \& Shabala, S. S. 2015, ApJ, 806, 59

Vincent, F. H., Paumard, T., Gourgoulhon, E., \& Perrin, G. 2011, CQGra, 28, 225011

Vlahakis, N., \& Königl, A. 2004, ApJ, 605, 656

Wang, F. Y., Yi, S. X., \& Dai, Z. G. 2014, ApJL, 786, L8

Werner, G. R., Uzdensky, D. A., Cerutti, B., Nalewajko, K., \& Begelman, M. C. 2016, ApJL, 816, L8

Wu, Q., Cao, X., Ho, L. C., \& Wang, D.-X. 2013, ApJ, 770, 31

Younsi, Z., Wu, K., \& Fuerst, S. V. 2012, A\&A, 545, A13

Zensus, J. A. 1997, ARA\&A, 35, 607 